\title{
Distributed Sensing of a Cantilever Beam and Plate using a Fiber Optic Sensing System
}

\author{
Patrick S. Heaney* and Thomas G. Ivanco ${ }^{\dagger}$ \\ NASA Langley Research Center, Hampton, VA, 23681 \\ Onur Bilgen \\ Rutgers University, Piscataway, NJ, 08854
}

\begin{abstract}
As the capabilities of Fiber Optic Sensing Systems continue to improve, their application to real-time distributed sensing for structural analysis and control of flexible systems is increasingly feasible. This paper will report experimental results on the use of a Fiber Optic Sensing System for static and dynamic shape estimation of a cantilever beam and plate. Demonstrating the use of this sensor technology in benchtop experiments is the first step in effectively incorporating fiber optic sensors in the Integrated Adaptive Wing Technology Maturation aeroelastic half-span wind tunnel model for real-time shape sensing and feedback for drag optimization, maneuver load alleviation, gust load alleviation, and flutter suppression control laws. The effectiveness of the sensing system will be analyzed and the application of these results to aeroelasticity experimentation will be discussed.
\end{abstract}

\section{Nomenclature}

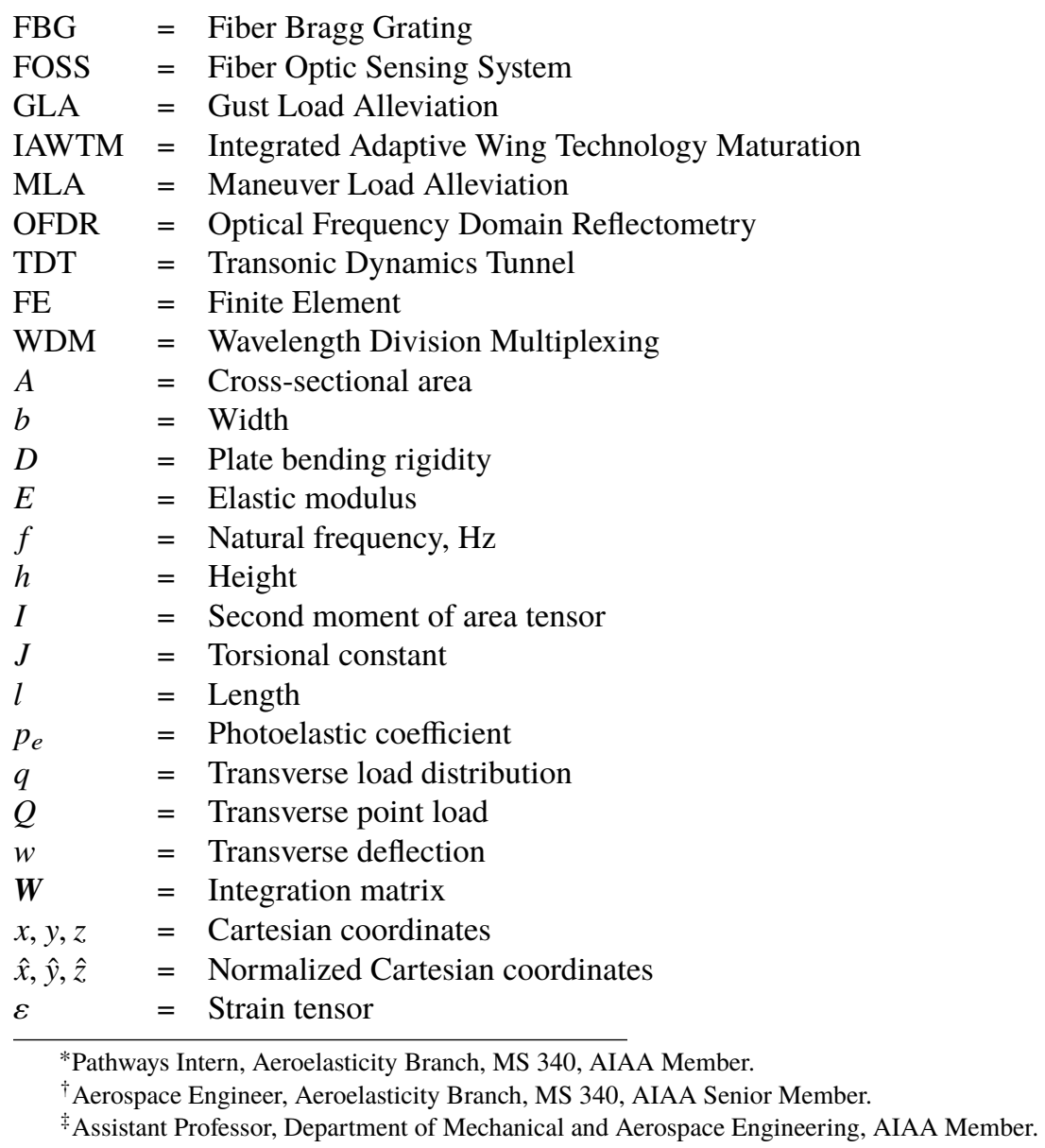




$\begin{array}{ll}\eta & =\text { Modal coordinate } \\ \lambda_{B} & =\text { Bragg wavelength } \\ \lambda_{i} & =\text { Natural frequency parameter } \\ v & =\text { Poisson's ratio } \\ \rho & =\text { Density } \\ \phi & =\text { Clamped-free beam mode shape } \\ \chi & =\text { Free-free beam mode shape } \\ \omega & =\text { Circular natural frequency, rad/s }\end{array}$

\title{
II. Introduction
}

\begin{abstract}
$\mathrm{A}^{\mathrm{n}}$ IRCRAFT wings have traditionally been designed to minimize structural flexibility and aeroelastic effects during flight in order to promote aircraft stability and performance. While this conventional design methodology seeks to maximize performance metrics in a specific operating range, its focus on structural inflexibility and static wing geometry does not support performance optimization across a broad range of operating conditions and results in significant increases in airframe weight [1-6]. By taking advantage of aeroelastic effects, rather than increasing structural stiffness in order to minimize them, structural weight can be reduced and the increased wing flexibility can be controlled to reduce drag and structural loads and to provide flight control [5, 7]. Control of wing flexibility, however, requires sensor technology that can estimate wing shape and vibration during flight for input to aeroelastic control laws. In order to meet this need for distributed sensing, a Fiber Optic Sensing System (FOSS) can provide real-time distributed strain measurements for calculation of wing shape and feedback for static and dynamic aeroelastic control.

The objectives of the current research are to demonstrate the use of FOSS for shape estimation (quasistatic measurements) and mode shape identification (dynamic measurements) during benchtop testing of a cantilever beam and plate. The focus is on real-time, dynamic measurement, with the goal of applying this sensing technology to active control of a flexible wing. The foundation developed in the experimental tests described here will enable the use of FOSS as a distributed sensor for the Integrated Adaptive Wing Technology Maturation (IAWTM) aeroelastic wing and the evaluation of its performance in closed-loop control of static and dynamic aeroelastic effects.

In the following sections of this paper, background is provided on aeroelastic shape control, FOSS, and the IAWTM project, and then a brief description of FOSS operating principles is given. Next, analytical models of beam and plate mechanics are introduced and experimental results for static and dynamic shape identification are reported for both the cantilever beam and plate. The paper concludes with an evaluation of FOSS as a distributed sensor and its application to aeroelastic experimentation.
\end{abstract}

\section{Background}

\section{A. Aeroelastic Shape Control}

Several research programs have demonstrated the feasibility of flexible wing designs that reduce structural weight while maintaining aircraft performance. In the Active Aeroelastic Wing (AAW) program, improved control power and reductions in drag and structural weight were shown for an actively controlled flexible wing utilizing leading edge control surfaces and favorable wing twist. The design techniques utilized in the AAW program were shown to reduce the aircraft takeoff gross weight by five to 20 percent [8]. Utilizing active controls and leading and trailing edge control surfaces, the Active Flexible Wing (AFW) program demonstrated the ability of a flexible wing to achieve desired load alleviation and flutter suppression while reducing structural weight [3,9]; similar results have also been shown for other airframes [6]. Researchers have predicted that a full implementation of the AFW concept would lead to a reduction in takeoff gross weight of 15 percent [6,9]. Developing the enabling technologies to support such flexible wing designs can thus lead to significant increases in performance.

\section{B. Fiber Optic Shape Sensing}

By providing distributed strain measurements which can be used for shape estimation, FOSS is one enabling technology for increased efficiency designs employing flexible structures. In comparison to conventional strain gauges, FOSS is lightweight and enables higher spatial resolution of the strain field. Two FOSS interrogation methods are frequently used: one type offers higher spatial resolution with lower frequency bandwidth and the other offers higher 
frequency bandwidth with lower spatial resolution. In this paper, the latter is utilized since the goal is to estimate the dynamic response of the structure at relatively high frequencies.

Several procedures have been developed to transform distributed strain measurements from FOSS into estimated deflection shapes. A common method, which is utilized in this paper, relies on estimating the curvature from strain measurements and then integrating the curvature twice to determine the deflection. Ko [10] described the implementation of this method and presented results, based on simulated strain measurements from finite element (FE) analysis, which validated the accuracy of the shape reconstruction for beams and for plates with multiple spanwise fibers. In order to construct the body's three-dimensional deformation field, estimated deflections along sensing fibers can be interpolated using an FE model to points in the body not instrumented with FOSS. This process is known as the inverse FE method [11-13]. Extending the previous shape reconstruction algorithm detailed by Ko, Pak [13] described a two-step method that included an FE model to interpolate the deformation for the entire structure based on strain measurements from parallel spanwise fibers. For various load cases, his method was accurate in reconstructing the deformation field in comparison to photogrammetry data. A number of additional experimental studies have been conducted comparing shape estimates based on FOSS with other sensing techniques (including photogrammetry), indicating relatively high accuracy depending on the shape sensing algorithm employed [14-19].

In addition to studies utilizing FOSS for static shape sensing, other researchers have studied its use for dynamic measurement and characterization [20,21] as well as active control. Several studies have demonstrated improved vibration suppression for control laws which incorporate distributed strain measurements based on FOSS in comparison to conventional point sensors [22-24]. As indicated by these benchtop studies, the distributed sensing capability of FOSS has the potential to enable improved controller performance for a variety of control objectives.

\section{Integrated Adaptive Wing Technology Maturation}

The IAWTM project is a NASA/Boeing joint effort with the objective of exploiting active controls to mitigate weight penalties typically incurred by very high aspect ratio wings. The IAWTM aircraft is a modern transport configuration based on the NASA Common Research Model [25], but with an increased aspect ratio of 13.5 compared to the nominal value of nine. IAWTM is complementary to and builds upon prior passive aeroelastic optimization efforts, such as tow-steered composite layup, that have been employed on this same high aspect ratio wing. In particular, active controls are sought for maneuver load alleviation (MLA), gust load alleviation (GLA), and flutter suppression while simultaneously achieving drag optimization for off-design conditions. These combined efforts are expected to further reduce structural weight beyond that possible solely through passive tailoring.

Following extensive analytical trade studies and control law development, the IAWTM project will culminate with a wind tunnel demonstration in the NASA Langley Transonic Dynamics Tunnel (TDT). FOSS will support the IAWTM test objectives by enabling sensing of the quasistatic wing deformation and dynamic mode shapes of the flexible wing during testing, and serving as input to the static and dynamic aeroelastic control laws to be tested. The potential for improved efficiency based on FOSS distributed sensing will be validated and the feasibility of incorporating FOSS in future aeroservoelastic tests will be evaluated.

\section{Fiber Optic Sensing System}

The operating principle behind FOSS is a coupled response between the optical and mechanical characteristics of an optical sensor. The optical sensor, known as a Fiber Bragg Grating (FBG), reflects a nominal wavelength of incident light, its Bragg wavelength, which is specified during manufacture. Changes in strain lead to proportional changes in the reflected wavelength according to Eq. (1),

$$
\frac{\Delta \lambda_{B}}{\lambda_{B}}=\left(1-p_{e}\right) \varepsilon,
$$

where $\lambda_{B}$ is the Bragg wavelength and $p_{e}$ is the photoelastic coefficient for the fiber. These changes can be detected using an optical interrogator. Although changes in temperature also cause changes in the Bragg wavelength, in this paper the temperature is assumed to be constant throughout the experimental measurements. Equation (1) can be used to calculate the strain at each sensor based on measured changes in the Bragg wavelength.

In Optical Frequency Domain Reflectometry (OFDR) interrogation, many FBGs manufactured at the same Bragg wavelength can be positioned in series along one optical fiber, and their individual changes in Bragg wavelength can be measured through digital signal processing algorithms. By tracking shifts in the peak reflected wavelengths, changes in the detected Bragg wavelength for each sensor can be mapped to corresponding changes in strain by Eq. (1). Although 
the OFDR interrogation method enables the multiplexing of hundreds of sensors at the same Bragg wavelength on a single fiber, its dynamic measurement capabilities are relatively low compared to conventional sensors. The maximum sampling rate for OFDR systems are generally less than $100 \mathrm{~Hz}$, with relatively high measurement latency. For a variety of applications, these measurement characteristics are sufficient to meet test objectives; for active control of gust loads or flutter suppression, however, the sampling rate must be higher.

In an alternate interrogation technique, known as Wavelength Division Multiplexing (WDM), the FBGs on one sensing fiber are manufactured at distinct nominal Bragg wavelengths. Each FBG occupies its own discrete region in wavelength space, and during an incident wavelength sweep the contribution of each FBG to the total measured response can be determined by tracking the movement of individual spectral peaks. This interrogation method is able to achieve significantly higher sampling rates than OFDR (on the order of $1 \mathrm{kHz}$ ). The tradeoff, however, is that fewer sensors can be etched on a single fiber due to the finite wavelength range of the laser's sweep, with most systems supporting approximately 40 sensors per fiber. For the IAWTM objectives, it was determined that 40 sensors per fiber would provide sufficiently dense measurements, so a higher sampling rate WDM system was chosen in order to provide feedback for both static and dynamic aeroelastic control laws. This is also the system that is used for the benchtop tests described in this paper.

\section{Beam Mechanics}

\section{A. Theoretical Model}

For a uniform beam with constant rectangular cross-section, the classical static governing equation for the transverse motion of the centerline is given by Eq. (2):

$$
E I_{y} \frac{d^{4} w(x)}{d x^{4}}=q(x)
$$

where $I_{y}=\iint_{A} z^{2} d A=b h^{3} / 12$ is the second moment of area for the cross section, $E I_{y}$ is the bending stiffness, $w(x)$ is the transverse deflection of the beam centerline, and $q(x)$ is the transverse load distribution. A schematic of the relevant dimensions for a beam with clamped-free boundary conditions is shown in Fig. 1.

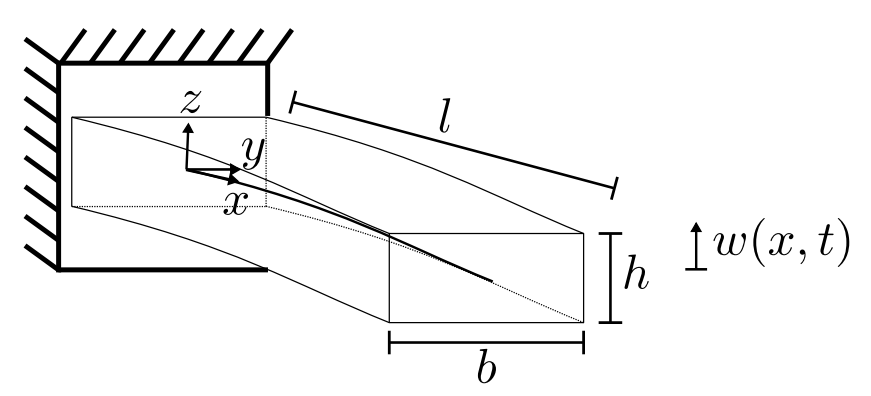

Fig. 1 Schematic of a cantilever beam in bending.

The assumptions of classical beam theory include: (1) the beam width and thickness are much smaller than its length, (2) the beam deflection is small, (3) the beam centerline does not stretch, (4) cross sections of the beam are rigid and remain normal to the beam centerline, and (5) rotational inertia and transverse shear are negligible.

Normalizing the longitudinal domain by the beam length $(\hat{x}=x / l)$, under the boundary conditions for a cantilever beam and a transverse load of $q(\hat{x})=Q \cdot \delta(1)$, where $Q$ is the point load applied at the tip, the static deflection solution is:

$$
w(\hat{x})=\frac{Q l^{3}}{6 E I_{y}} \hat{x}^{2}(3-\hat{x}) .
$$

Differentiating twice with respect to $x$ yields the curvature:

$$
\frac{d^{2} w(\hat{x})}{d x^{2}}=\frac{1}{l^{2}} \frac{d^{2} w(\hat{x})}{d \hat{x}^{2}}=\frac{Q l}{E I_{y}}(1-\hat{x}) .
$$

The static deflection and curvature shapes, normalized to have a maximum value of 1, are shown in Fig. 2. 

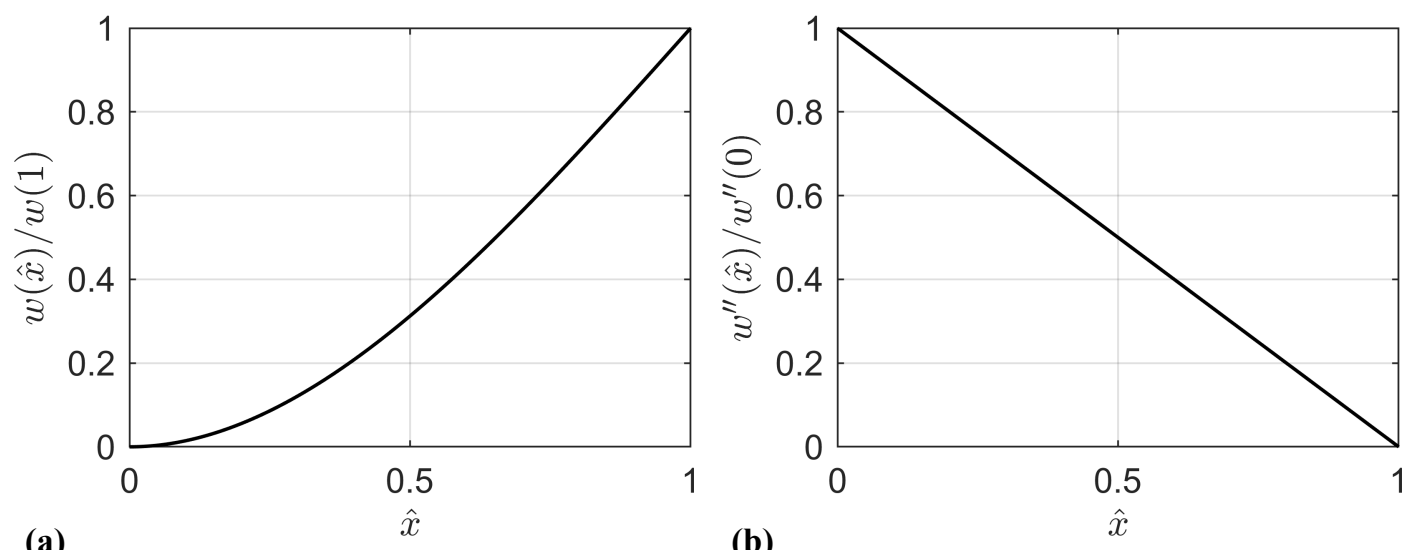

Fig. 2 (a) Normalized static deflection and (b) normalized static curvature of a cantilever beam.

The surface strain on the top of the beam, which is linearly related to the beam's curvature, is given by:

$$
\varepsilon_{x x}(\hat{x})=-\frac{h}{2 l^{2}} \cdot \frac{d^{2} w(\hat{x})}{d \hat{x}^{2}} .
$$

Distributed strain measurements along the upper surface of a beam with a static tip load, therefore, should closely follow the linearly decreasing profile shown in Fig. 2(b). This will be demonstrated in the experimental section using FOSS.

The dynamic classical beam equation of motion for undamped, free vibration is given by:

$$
\rho A \frac{\partial^{2} w(x, t)}{\partial t^{2}}+E I_{y} \frac{\partial^{4} w(x, t)}{\partial x^{4}}=q(x, t),
$$

where $\rho$ is the density and $A$ is the cross-sectional area of the beam. The corresponding cantilever boundary conditions are given in Eq. (7), where the beam is clamped at $\hat{x}=0$ and free at $\hat{x}=1$ :

$$
\begin{array}{lll}
w(0, t) & =0 & \left.\frac{\partial^{2} w(\hat{x}, t)}{\partial \hat{x}^{2}}\right|_{\hat{x}=1}=0 \\
\left.\frac{\partial w(\hat{x}, t)}{\partial \hat{x}}\right|_{\hat{x}=0}=0 & \left.\frac{\partial^{3} w(\hat{x}, t)}{\partial \hat{x}^{3}}\right|_{\hat{x}=1}=0 .
\end{array}
$$

Using the separation of variables method and expressing the solution to Eq. (6) as $w(\hat{x}, t)=\phi(\hat{x}) \eta(t)$, where $\phi(\hat{x})$ is the mode shape and $\eta(t)$ is the modal coordinate, the spatial solution under the boundary conditions given in Eq. (7) yields the characteristic equation:

$$
1+\cos \lambda_{r} \cosh \lambda_{r}=0
$$

where the quantity $\lambda_{r}$, known as the natural frequency parameter, is defined as $\lambda_{r}=\left(\sqrt[4]{\rho A l^{4} \omega_{r}^{2} / E I_{y}}\right)$ and $\omega_{r}$ is the circular natural frequency. Expressing this relationship for $\omega_{r}$, the corresponding natural frequencies for a cantilever beam are given by:

$$
\omega_{r}=\left(\sqrt{\frac{E I_{y}}{\rho A l^{4}}}\right) \lambda_{r}^{2}
$$

Solutions to Eq. 8 can then be substituted to yield the system natural frequencies.

The spatial solution also yields the mode shapes of the cantilever beam:

$$
\phi_{r}(\hat{x})=\cos \lambda_{r} \hat{x}-\cosh \lambda_{r} \hat{x}-\left(\frac{\cos \lambda_{r}+\cosh \lambda_{r}}{\sin \lambda_{r}+\sinh \lambda_{r}}\right)\left(\sin \lambda_{r} \hat{x}-\sinh \lambda_{r} \hat{x}\right) .
$$

The first four of these mode shapes have been plotted in Fig. 3 where they have been normalized to have a maximum absolute value of 1 . The curvature profiles for these mode shapes $\left(\phi_{r}^{\prime \prime}(\hat{x})\right)$ have also been plotted in Fig. 3(b) with the 
same normalization. As previously discussed for the static solution, the surface strain on the top of the beam is linearly related to the beam curvature, so distributed strain measurements from a FOSS fiber bonded to the upper surface of a dynamically excited cantilever beam should correspond with the shape of the normalized curvature profiles shown here for each mode.
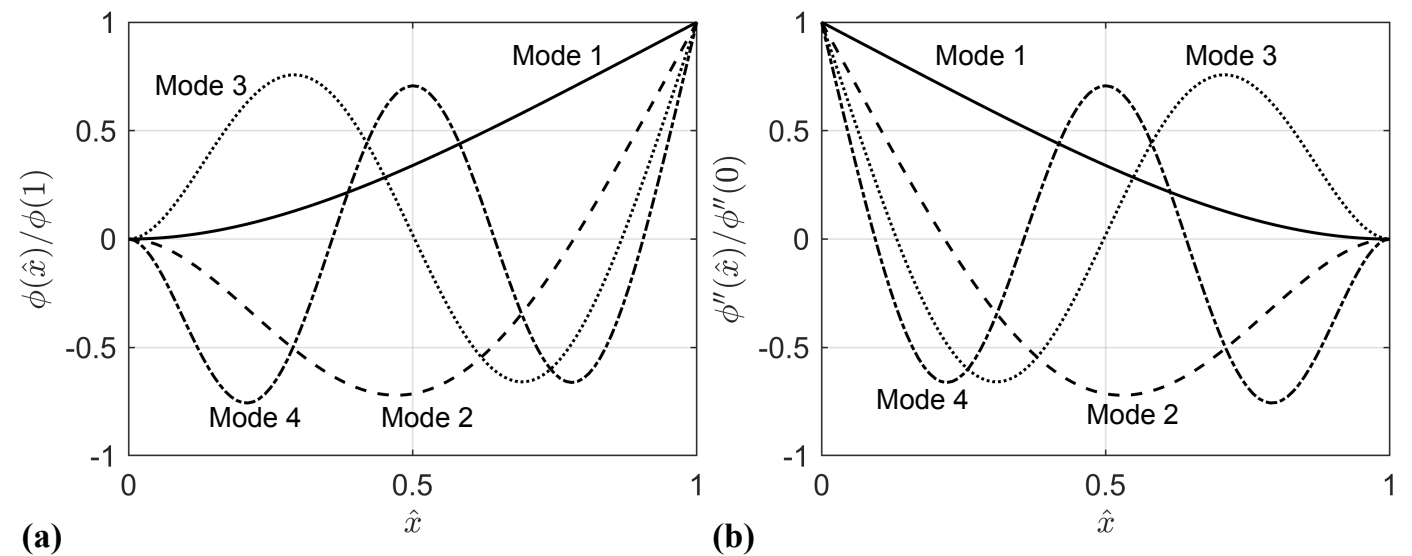

Fig. 3 (a) Normalized dynamic mode shapes and (b) normalized dynamic curvature shapes of a cantilever beam.

\section{B. Shape Estimation from Strain Field}

If the strain field is a known continuous function, the curvature can be directly determined from Eq. (5) and then integrated twice to yield the cantilevered beam deflection profile based on zero integration constants at the root. Since a finite number of FBGs can be etched on a single fiber, however, the FOSS strain output is a vector of discrete strain measurements. Several methods can be used to numerically integrate these discrete strain measurements in order to estimate the deflection profile for the beam. The Newmark- $\beta$ method [26] is a single step method used in structural dynamics for integration of known accelerations to determine velocities and displacements. It can be applied here to the known curvature estimates, computed from the strain field using Eq. (5), to yield the slopes and deflections at the FOSS strain stations. Using the common parameters of the Newmark method ( $\alpha=1 / 2$ and $\beta=1 / 4)$, the slope and deflection at station $i+1$ are given by Eq. (11):

$$
\begin{aligned}
& w_{i+1}^{\prime}=w_{i}^{\prime}+\frac{\Delta x}{2} \cdot\left(w_{i}^{\prime \prime}+w_{i+1}^{\prime \prime}\right) \\
& w_{i+1}=w_{i}+\Delta x \cdot w_{i}^{\prime}+\frac{\Delta x^{2}}{4} \cdot\left(w_{i}^{\prime \prime}+w_{i+1}^{\prime \prime}\right),
\end{aligned}
$$

where $\Delta x$ is the distance between strain stations and ' indicates differentiation with respect to $x$. Similar numerical techniques for determining structural deformation based on strain measurements have been used by other researchers [10, $17]$.

Assuming that the discrete strain measurements either span the domain of the beam or are extrapolated to do so, one way to implement the numerical integration based on Eq. (11) uses matrices as the "integrators". For the boundary conditions of the cantilever beam, the $i+1$ slope estimate is:

$$
w_{i+1}^{\prime}=\frac{\Delta x}{2}\left(w_{0}^{\prime \prime}+2 \sum_{k=1}^{i} w_{k}^{\prime \prime}+w_{i+1}^{\prime \prime}\right) \text {. }
$$


The resulting equations for $N$ positions, including the beam root, can be arranged as an $N \times 1$ vector:

$$
\boldsymbol{w}^{\prime}=\left\{\begin{array}{c}
w_{0}^{\prime} \\
w_{1}^{\prime} \\
w_{2}^{\prime} \\
w_{3}^{\prime} \\
\vdots \\
w_{N-1}^{\prime}
\end{array}\right\}=\frac{\Delta x}{2}\left[\begin{array}{cccccc}
0 & 0 & 0 & 0 & 0 & \cdots \\
1 & 1 & 0 & 0 & 0 & \ldots \\
1 & 2 & 1 & 0 & 0 & \ldots \\
1 & 2 & 2 & 1 & 0 & \ldots \\
\vdots & & & & \ddots & \vdots \\
1 & 2 & 2 & \ldots & 2 & 1
\end{array}\right]\left\{\begin{array}{c}
w_{0}^{\prime \prime} \\
w_{1}^{\prime \prime} \\
w_{2}^{\prime \prime} \\
w_{3}^{\prime \prime} \\
\vdots \\
w_{N-1}^{\prime \prime}
\end{array}\right\}=\boldsymbol{W} \boldsymbol{w}^{\prime \prime} .
$$

The matrix $\boldsymbol{W}$ can be interpreted as the "integration matrix", which can be used to determine the slope vector from the curvature vector. It turns out that the exact same pattern holds for the deflection estimates based on the slope vector, such that:

$$
w=W w^{\prime}=W^{2} w^{\prime \prime},
$$

where the matrix $\boldsymbol{W}$ is applied twice to determine the deflection vector $\boldsymbol{w}$ from the curvature vector $\boldsymbol{w}^{\prime \prime}$.

An additional numerical integration strategy is to interpolate the acquired discrete data between the strain stations using a polynomial in order to increase the number of integration points. One such scheme known as a cubic spline uses piecewise cubic polynomials for interpolation, which ensures that the curvature of the spline is continuous throughout the interpolation region. This method has previously been used in various formulations to assess its effectiveness in shape reconstruction [16]. The Newmark numerical integration scheme can then be applied to the interpolated data to determine the beam deflection.

One note on applying this numerical integration strategy that should be emphasized is that the discrete strain measurements must span the domain of the beam, i.e., measurements at the root and tip must be available in order to apply this technique. Practically speaking, neither estimate is easy to make using bonded sensing fibers. Instead, for the experimental cases in this paper the discrete strain measurements are always extrapolated using a cubic spline to yield estimates spanning the beam length.

With the known deflection and strain profiles for a tip loaded cantilever beam, simulated FOSS measurements can be computed from Eq. (5) using the static curvature profile given by Eq. (4) at discrete strain stations along the beam domain. The material and geometric properties of the beam to be analyzed are given in Table 1, which correspond to the beam properties for the experimental specimen described later.

Table 1 Material and geometric properties of the beam specimen.

\begin{tabular}{lcl}
\hline Parameter & Variable & Value \\
\hline Material & & Aluminum 6061 \\
Mass, Total & $m$ & $157 \mathrm{~g}$ \\
Density & $\rho$ & $2640 \mathrm{~kg} / \mathrm{m}^{3}$ \\
Elastic modulus & $E$ & $68.9 \mathrm{GPa}[27]$ \\
Poisson's ratio & $v$ & $0.35[27]$ \\
Length, Total & & $488 \mathrm{~mm}$ \\
Length, Free & $l$ & $450 \mathrm{~mm}$ \\
Width & $b$ & $38.1 \mathrm{~mm}$ \\
Height & $h$ & $3.20 \mathrm{~mm}$ \\
\hline
\end{tabular}

For the simulated data, the curvature/strain is calculated based on an applied tip load of $1 \mathrm{~N}$. The first strain measurement is calculated at $20 \mathrm{~mm}$ from the root of the beam and subsequent measurements are calculated at $20 \mathrm{~mm}$ intervals along the beam's span. This arrangement is based on the bonding scheme for the benchtop cantilever beam tested in the experimental section, which is instrumented with a fiber with FBGs $20 \mathrm{~mm}$ on center. These simulated measurements are then integrated using the schemes described above and compared to the analytical deflection profile given by Eq. (3).

Two cases are compared: one without interpolation and the second with a cubic spline. The cubic spline used for the second case interpolates the simulated strain measurements every $5 \mathrm{~mm}$, corresponding to a quadrupling of the 
strain measurements used for integration. As previously noted, the root and tip measurements must be extrapolated in the implementation of this integration scheme. For the interpolation case, the same $5 \mathrm{~mm}$ spacing is used in the extrapolation regions from the root to the first sensor and from the last sensor to the tip. In the integration case without interpolation, only the root and tip measurements are extrapolated.

The integrated deflection profiles using the simulated data is shown in comparison to the analytical deflection in Fig. 4 for exclusive Newmark integration and Newmark integration of the cubic spline. Qualitatively, integration after the cubic spline leads to a result that is almost identical to the analytical (an error of only $0.003 \%$ at the tip) while the exclusive Newmark integration of the simulation strain is significantly farther from the analytical (an error of more than $3 \%$ at the tip).

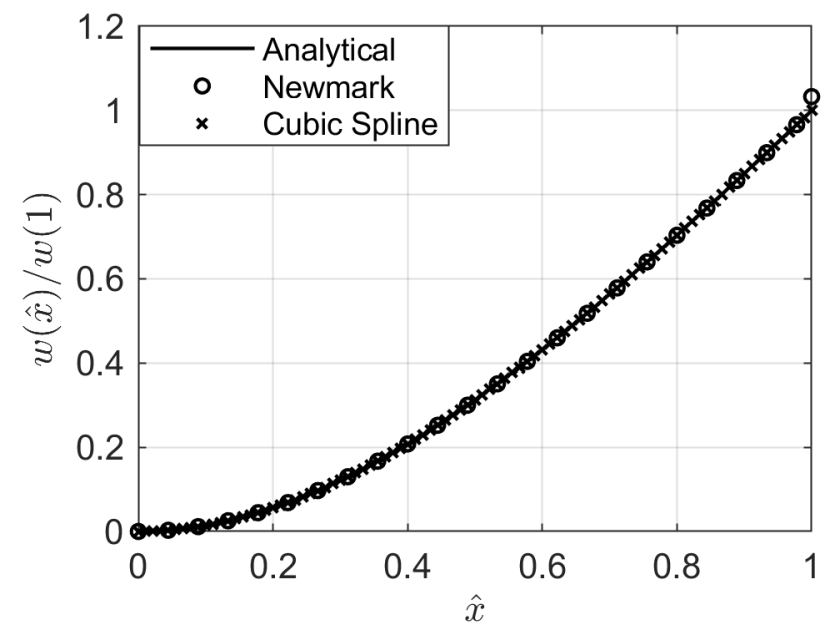

Fig. 4 Static deflection of the cantilever beam for a point tip load based on different integration algorithms of simulated FOSS strain data.

\section{Mode Identification from Strain Field}

The same integration methods described for the static case can also be applied for dynamic mode shape identification. Here, simulated FOSS strain data is computed as previously described using Eq. (5) with the mode shapes given by Eq. (10) to compute the strain for the first four modes. The same Newmark integration scheme, with and without cubic spline interpolation, is then conducted and the results compared to the analytical mode shapes in Fig. 5. 


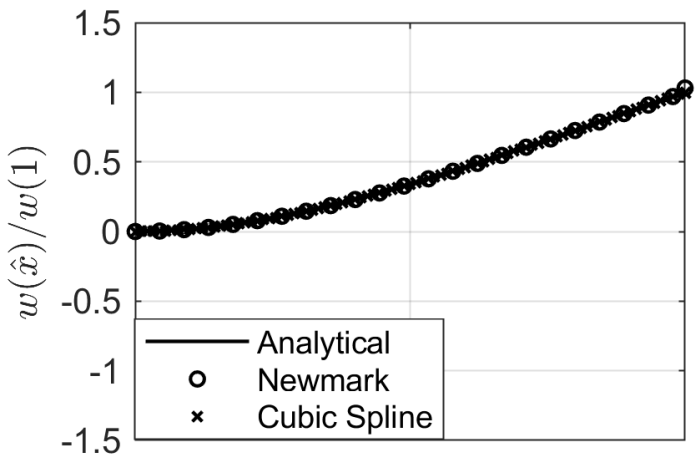

(a) Mode 1

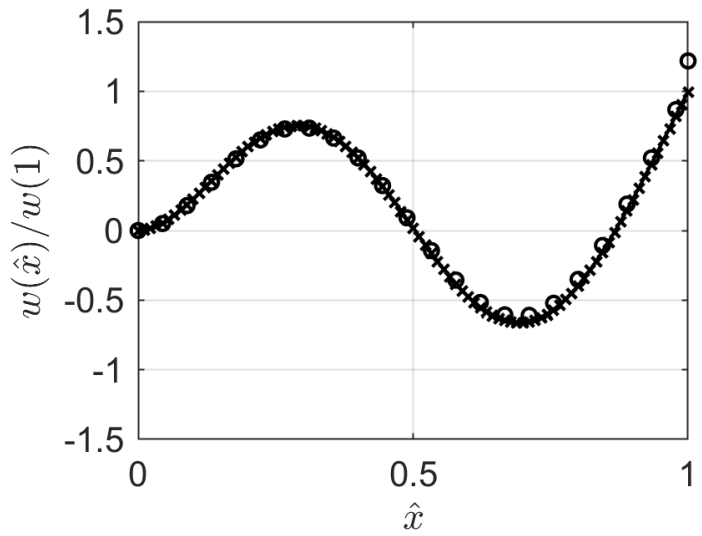

(c) Mode 3

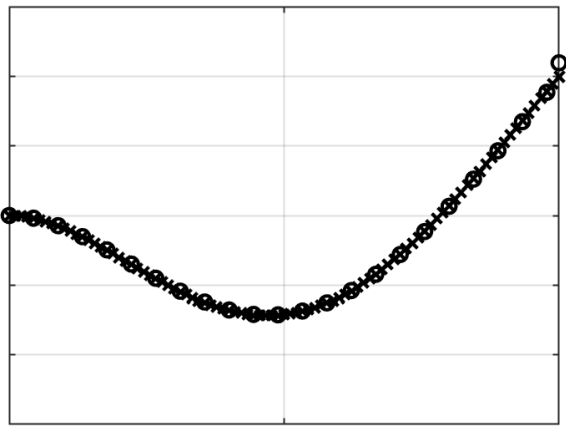

(b) Mode 2

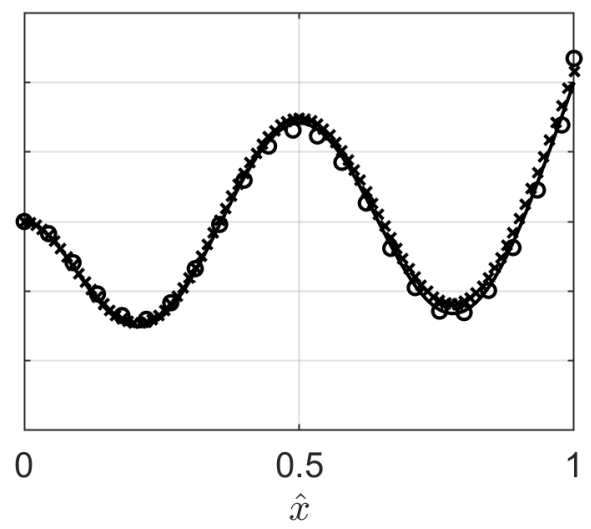

(d) Mode 4

Fig. 5 Mode shapes of the cantilever beam based on different integration algorithms of simulated FOSS strain data.

To compare the results of these integration schemes with the analytical mode shapes, the maximum relative errors in shape for both integration methods, which occur at the beam tip, are reported in Table 2.

Table 2 Mode shape tip deflection error for different integration algorithms of simulated FOSS strain data.

\begin{tabular}{lcccc}
\hline Method & Mode 1 & Mode 2 & Mode 3 & Mode 4 \\
\hline Newmark & $3.0 \%$ & $9.6 \%$ & $21.8 \%$ & $17.2 \%$ \\
Cubic Spline plus Newmark & $0.002 \%$ & $0.002 \%$ & $0.9 \%$ & $7.8 \%$ \\
\hline
\end{tabular}

As for the integration of simulated static strain data, interpolating the discrete strain measurements before integration yields results that are significantly more accurate than exclusive integration of the simulated strain measurements. Based on these results, cubic spline interpolation will be implemented for static and dynamic shape estimation in the experimental section.

\section{Plate Mechanics}

\section{A. Theoretical Model}

As with the beam, several assumptions are utilized in classical plate theory: (1) the plate thickness is much smaller than its length and width, (2) the plate deflection is small, (3) the plate midplane does not stretch, (4) cross sections of the plate are rigid and remain to normal to the plate midplane, and (5) rotational inertia and transverse shear are 
negligible. The plate motion is assumed to be a function only of the $x$ - and $y$-coordinates and time, and thus, can be fully described by the deformation of the midplane shown in gray in Fig. 6.

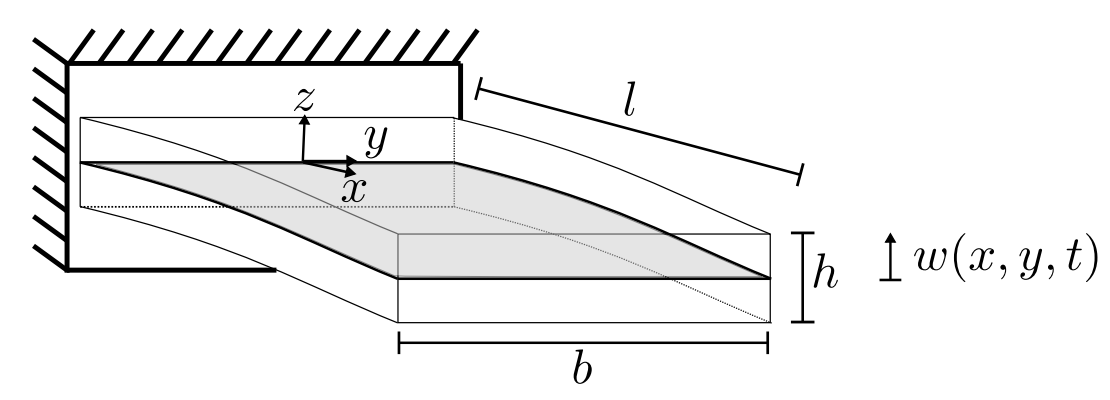

Fig. 6 Schematic of a cantilever plate.

The classical plate equation of motion for undamped free vibration is given by:

$$
\rho h \frac{\partial^{2} w(x, y, t)}{\partial t^{2}}+D \nabla^{4} w(x, y, t)=q(x, y, t),
$$

where $D=E h^{3} / 12\left(1-v^{2}\right)$ is the bending rigidity of the plate, $v$ is Poisson's ratio, and $\nabla^{4}(\cdot)=\left(\partial_{x x x x}+\partial_{x x y y}+\partial_{y y y y}\right)(\cdot)$ is the biharmonic operator. The boundary conditions for a rectangular plate oriented with sides parallel to the $x$ - and $y$-axes are:

$$
\begin{aligned}
M_{x} \delta\left(\partial_{x} w\right) & =0 \\
M_{y} \delta\left(\partial_{y} w\right) & =0 \\
\left(V_{x}+\partial_{y} M_{x y}\right) \delta w & =0 \\
\left(V_{y}+\partial_{x} M_{x y}\right) \delta w & =0,
\end{aligned}
$$

where $M$ and $V$ are moments and shear forces.

Following the approximate method described and utilized extensively by others [28-32], the Rayleigh-Ritz method can be used to determine an upper bound of the natural frequencies for plate structures after specifying candidate functions to describe the mode shapes. For rectangular plates, one of the more useful sets of candidate functions are those composed of products of the beam functions corresponding to the boundary conditions at opposite sides of the plate. The total plate response is then given by the summation of these beam products:

$$
w(\hat{x}, \hat{y})=\sum_{r} \sum_{s} C_{r s} \phi_{r}(\hat{x}) \chi_{s}(\hat{y})
$$

where $\phi_{r}$ and $\chi_{s}$ are the beam mode shapes corresponding to the boundary conditions in the $\hat{x}$ - and $\hat{y}$-directions, and $C_{r s}$ are coefficients describing the contribution of each candidate mode shape to the total response. For a given number $n$ of beam mode shapes in each of the coordinate directions, the Rayleigh-Ritz method can be used to yield estimated eigenvalues, $\lambda_{i}$, for the $n^{2}$ modes of the plate, which are approximated by corresponding products of those beam functions. The natural frequencies of the plate can then be expressed as:

$$
\omega_{i}=\left(\sqrt{\frac{D}{\rho h l^{4}}}\right) \lambda_{i}^{2} .
$$

For the clamped-free plate, $\phi_{r}$ are the clamped-free beam mode shapes given by Eq. (10) and $\chi_{s}$ are the free-free beam mode shapes. The flexible free-free beam modes are:

$$
\chi_{s}(\hat{y})=\sin \lambda_{s} \hat{y}-\sinh \lambda_{s} \hat{y}-\left(\frac{\sinh \lambda_{s}-\sin \lambda_{s}}{\cosh \lambda_{s}-\cos \lambda_{s}}\right)\left(\cos \lambda_{s} \hat{y}+\cosh \lambda_{s} \hat{y}\right),
$$

where $\lambda_{s}$ are the solutions to the characteristic equation:

$$
1-\cos \lambda_{s} \cosh \lambda_{s}=0 .
$$


In addition to these flexible modes, the free-free beam also has two rigid body modes: (1) pure translation, which has a mode shape described by $\chi_{1}^{r}(\hat{y})=1$, and (2) pure rotation, which has a mode shape described by $\chi_{2}^{r}(\hat{y})=1-2 \hat{y}$. Although the products of beam functions yield reasonably accurate models for the mode shapes and predictions for the natural frequencies of a cantilever plate, it should be noted that they do not exactly meet the free edge boundary conditions.

Using the Rayleigh-Ritz method based on products of beam functions, the frequency parameters $\lambda_{i}$ are computed here for the plate specimen studied in this paper. Similar results have been tabulated in previous works for a range of boundary conditions and aspect ratios [30,31]. The material and geometric properties of the rectangular cantilever plate to be analyzed are given in Table 3, which correspond to the properties for the experimental plate specimen described later.

Table 3 Material and geometric properties of the plate specimen.

\begin{tabular}{lcl}
\hline Parameter & Variable & Value \\
\hline Material & & Aluminum 6061 \\
Mass, Total & $m$ & $3.06 \mathrm{~kg}$ \\
Density & $\rho$ & $2730 \mathrm{~kg} / \mathrm{m}^{3}$ \\
Elastic modulus & $E$ & $68.9 \mathrm{GPa}[27]$ \\
Poisson's ratio & $v$ & $0.35[27]$ \\
Length, Total & & $860 \mathrm{~mm}$ \\
Length, Free & $l$ & $800 \mathrm{~mm}$ \\
Width & $b$ & $406 \mathrm{~mm}$ \\
Height & $h$ & $3.20 \mathrm{~mm}$ \\
Aspect Ratio $(l / b)$ & & 1.97 \\
\hline
\end{tabular}

In Fig. 7, the convergence of the frequency parameters as the number of candidate modes increases is shown. At each iteration, an additional candidate mode shape is added in both the $\hat{x}$ - and $\hat{y}$-directions and the eigenproblem solved to determine the estimated frequency parameters. As the number of candidate mode shapes increase, each frequency parameter is converging from above toward its final estimated value $\lambda_{i, f}$.

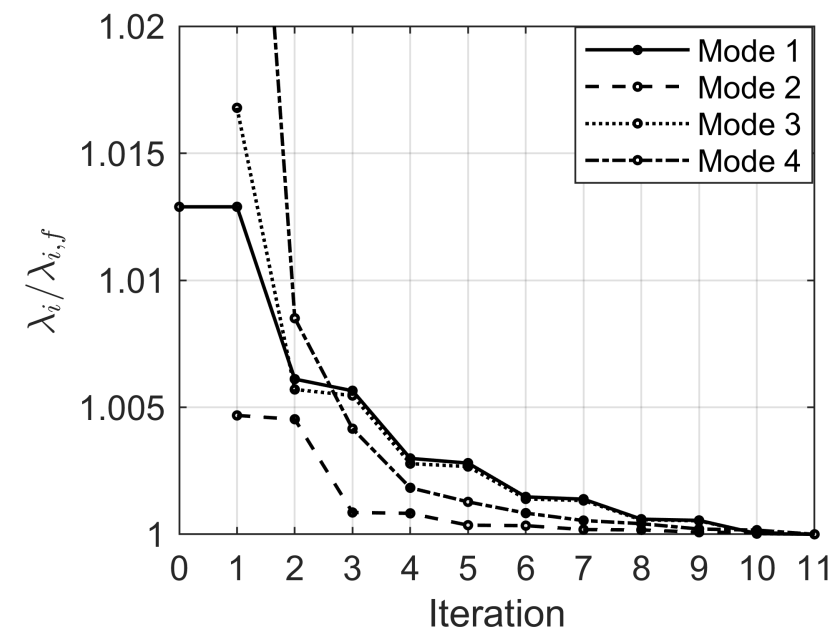

Fig. 7 Convergence of the frequency parameters $\lambda_{i}$ for the first four modes of the cantilever rectangular plate with $l / b=1.97$ and $v=0.35$ based on the Rayleigh-Ritz method and products of beam functions. The ratio $\lambda_{i} / \lambda_{i, f}$ indicates the percent difference between the iteration value and the final value of the corresponding frequency parameter. 
The final frequency parameters for this plate configuration are given in Table 4, where the corresponding mode shapes based on products of beam functions are also specified.

Table 4 Frequency parameters and approximate mode shapes for the cantilever plate with $l / b=1.97$ and $v=0.35$ based on the Rayleigh-Ritz method and products of beam functions.

\begin{tabular}{clcl}
\hline Mode & Description & $\lambda_{i}$ & Mode Shape \\
\hline 1 & Bending 1 & 1.8512 & $\phi_{1}(\hat{x}) \chi_{1}^{r}(\hat{y})=\phi_{1}(\hat{x})$ \\
2 & Torsion 1 & 3.7640 & $\phi_{1}(\hat{x}) \chi_{2}^{r}(\hat{y})=\phi_{1}(\hat{x})(1-2 \hat{y})$ \\
3 & Bending 2 & 4.6166 & $\phi_{2}(\hat{x}) \chi_{1}^{r}(\hat{y})=\phi_{2}(\hat{x})$ \\
4 & Torsion 2 & 6.8161 & $\phi_{2}(\hat{x}) \chi_{2}^{r}(\hat{y})=\phi_{2}(\hat{x})(1-2 \hat{y})$ \\
\hline
\end{tabular}

The FE mode shapes for the plate are also determined in MSC NASTRAN 2018.0 [33] using SOL 103 (normal modes analysis). A comparison of the approximate mode shapes based on products of beam functions to the FE mode shapes is shown in Fig. 8, where the shapes are scaled to have the same absolute maximum displacement.

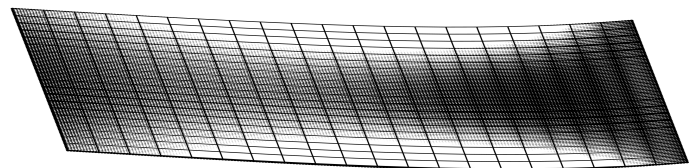

(a) Mode 1, Bending 1

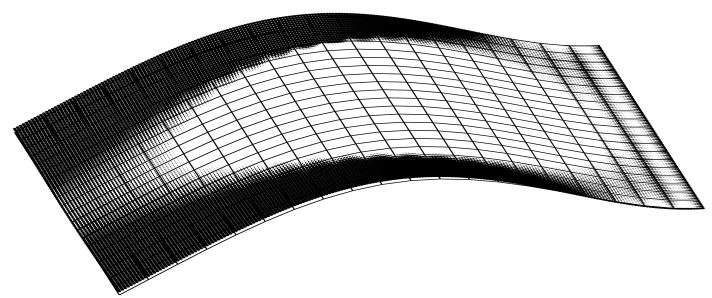

(c) Mode 3, Bending 2

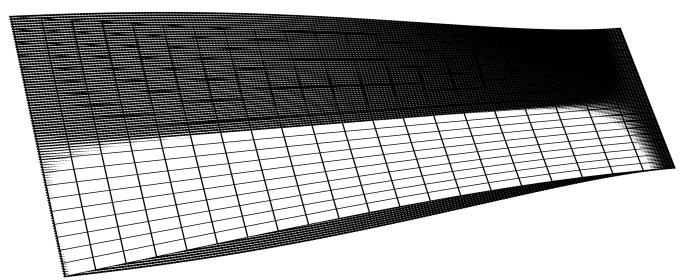

(b) Mode 2, Torsion 1

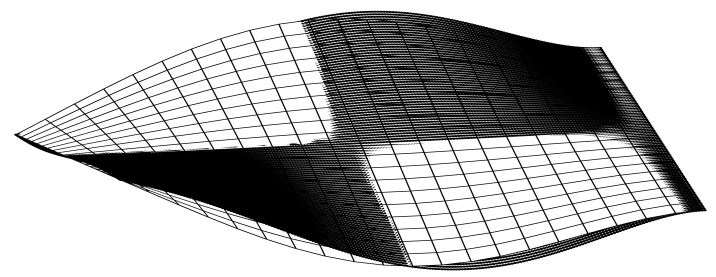

(d) Mode 4, Torsion 2

Fig. 8 Comparison of mode shapes for the cantilever rectangular plate with $l / b=1.97$ and $v=0.35$ determined by products of beam functions (grids) and $\mathrm{FE}$ analysis (dots). The clamped edge is on the right.

For all the modes presented, the general character of the shapes are the same for both techniques. However, it is also clear that the approximate mode shapes based on products of beam functions (plotted as gridded surfaces), do not perfectly account for the boundary conditions along the free edges. In particular, the increased stiffness of these approximate mode shapes compared to the FE shapes can be seen along the plate edges.

\section{B. Twist Rate Estimation from Strain Field}

A procedure for estimating the shape of a deformed cantilever plate based on the strain field will be described in this section; similar formulations have previously been described [13, 14, 34]. Consider the unprimed and primed axes shown in Fig. 9. 


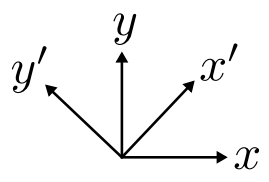

Fig. 9 Reference coordinate axes for shear and axial strain.

The strain in the direction of the rotated $x^{\prime}$-axis can be determined by the strain in the unrotated axes using the transformation equation for strain [28]:

$$
\varepsilon_{x^{\prime} x^{\prime}}=a_{x^{\prime} x}^{2} \varepsilon_{x x}+2 a_{x^{\prime} x} a_{x^{\prime} y} \varepsilon_{x y}+a_{x^{\prime} y}^{2} \varepsilon_{y y}=\frac{1}{2}\left(\varepsilon_{x x}+\varepsilon_{y y}\right)+\varepsilon_{x y},
$$

where the direction cosines between the two frames are $a_{x^{\prime} x}=a_{x^{\prime} y}=\sqrt{2} / 2$ for a 45 -deg rotation. For cylindrical bending about the $y$-axis (i.e., $\varepsilon_{y y}=\varepsilon_{x y}=0$ ), the axial strain in a fiber oriented at 45 -deg from the $x$-direction, $\varepsilon_{x^{\prime} x^{\prime}}$, is thus equal to half the bending strain $\varepsilon_{x x}$, and for pure torsion (i.e., the bending strains are $\varepsilon_{x x}=\varepsilon_{y y}=0$ ), $\varepsilon_{x^{\prime} x^{\prime}}$ is equal to the shear strain $\varepsilon_{x y}$. Thus, under pure torsion a fiber oriented at 45-deg can be used to determine the shear strain at that point.

For a narrow rectangular cross section, using the Prandtl stress function it can be shown $[28,35]$ that far from the narrow ends of the cross section the shear strain is given by:

$$
\varepsilon_{x y}=-\alpha z,
$$

where $\alpha$ is the twist rate. For pure torsional modes, since the shear strain is equal to $\varepsilon_{x^{\prime} x^{\prime}}$, the twist rate $\alpha$ can be determined directly based on measurements of axial strain in the $x^{\prime}$-direction:

$$
\alpha=-\frac{1}{z} \varepsilon_{x^{\prime} x^{\prime}} .
$$

For the case of general motion, estimating the twist rate by Eqn. (22) requires measurements of the normal strains in three directions $\left(x, y\right.$, and $\left.x^{\prime}\right)$ to compute the shear strain by Eqn. (21).

Based on this result, the same numerical integration strategies described above for estimating the transverse deflection can be used to estimate the twist distribution from distributed measurements of the twist rate. To illustrate one layout that incorporates strain measurements in the rotated $x^{\prime}$-direction, FE data are computed for three simulated spanwise fiber runs on the rectangular plate with properties specified in Table 3: (1) $\varepsilon_{x x}$ along the leading edge, (2) $\varepsilon_{x^{\prime} x^{\prime}}$ along the midchord, and (3) $\varepsilon_{x x}$ along the trailing edge. This is the fiber layout implemented for the experimental tests described later. These strain distributions are shown in Fig. 10 for the first four modes. 


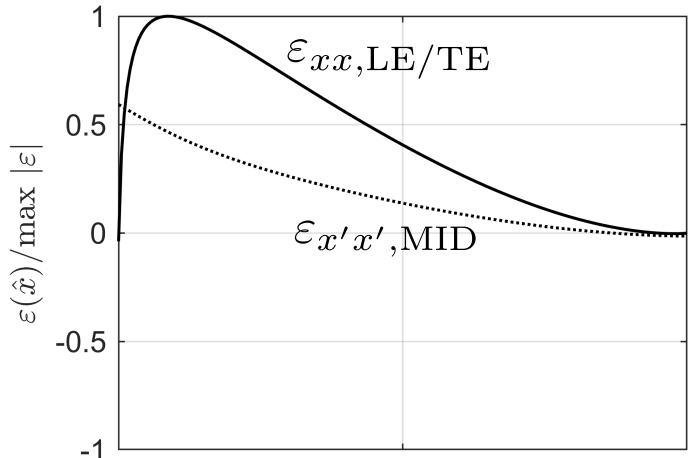

(a) Mode 1, Bending 1

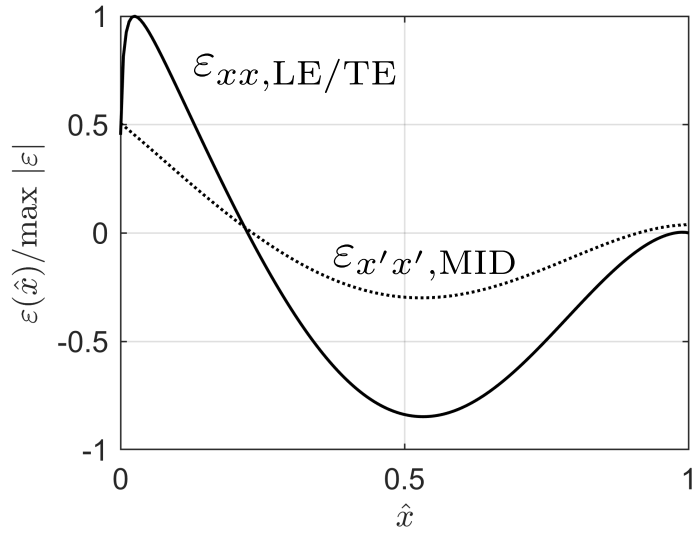

(c) Mode 3, Bending 2

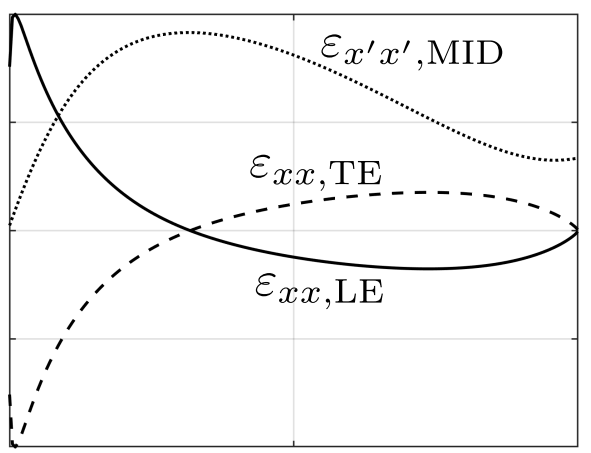

(b) Mode 2, Torsion 1

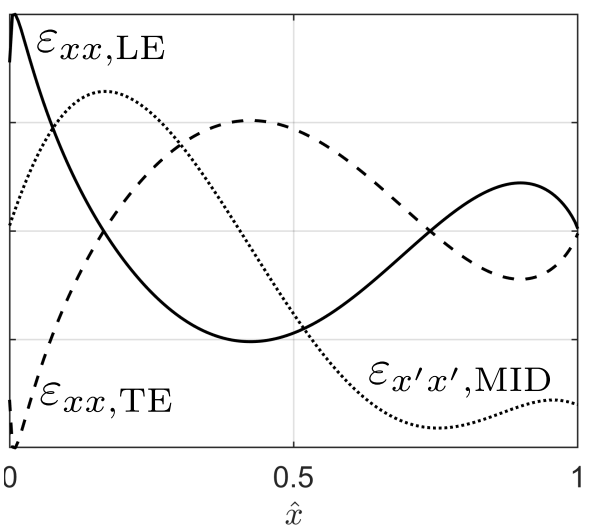

(d) Mode 4, Torsion 2

Fig. 10 Normal strain in the $x$-direction along the leading and trailing edges and in the $x^{\prime}$-direction along the midchord for the first four modes of the cantilever rectangular plate with $l / b=1.97$ and $v=0.35$ based on FE analysis.

The qualitative participation of each simulated fiber in a particular mode can be observed in these plots. Modes 1 and 3 , for example, are primarily bending modes and show identical responses for the two bending fibers (on the leading and trailing edges), which are approximately the same shape as the analytical curvatures for the first and second beam bending modes. As expected from the strain transform equation (Eq. (21)), the strain response for the midchord fiber is approximately half of the normal strain for the fibers oriented in the $x$-direction for Modes 1 and 3.

Modes 2 and 4, on the other hand, are somewhat more complex. The expected response of the midchord fiber for these modes, based on the products of beam functions given in Table 4, can be shown to correspond to the curvature shapes of the first two beam bending modes. A comparison of the shape in Fig. 10(b) for $\varepsilon_{x^{\prime} x^{\prime}, \mathrm{MID}}$ to that of $\varepsilon_{x x, \mathrm{LE}}$ in Fig. 10(a) shows a loose correspondence. A similar approximate correspondence can be seen in comparing the shape in Fig. 10(d) for $\varepsilon_{x^{\prime} x^{\prime}, \mathrm{MID}}$ to that of $\varepsilon_{x x, \mathrm{LE}}$ in Fig. 10(c). The major difference for these cases is that the tip boundary condition for the midchord fiber under torsion (which is proportional to the plate's twist rate) is not actually zero, so the products of beam functions do not perfectly satisfy the plate boundary conditions.

Several additional observations for modes 2 and 4 are also of interest: (1) the response of the midchord twist rate fiber, $\varepsilon_{x^{\prime} x^{\prime}, \mathrm{MID}}$, is of the same magnitude as the bending fibers, (2) the bending fibers respond in opposite directions to each other, and (3) the bending responses correspond approximately to the strain shapes of its preceding bending mode. The first two observations here imply two methods to identify the twisting mode shapes. Either the response of the midchord twist rate fiber can be used or the responses of the leading and trailing edge fibers can be compared. This second method has been used by Pak [13], where multiple longitudinally oriented fibers are used to approximate the deformation along each slice of a plate, which are then used to reconstruct the deformation of the entire structure. 


\section{Experimental Procedures and Results}

Following the development based on simulated strain data in the previous two sections, the integration scheme is implemented here for experimental data. The estimated tip displacement is then compared with experimental measurements for the static cases, and the estimated mode shapes are compared with the theoretical results.

The interrogator used here is the Micron Optics si-155, which supports 4 channels of sensing fibers and a sampling rate of $1 \mathrm{kHz}$. The sensor latency, measured in comparison with a collocated strain gauge, is approximately $0.65 \mathrm{~ms}$. Each sensing fiber utilized in this paper is manufactured by FBGS and is etched with 39 FBGs located $20 \mathrm{~mm}$ center-to-center. The fibers are attached to the beam and plate using Hysol EA 9394, a two part aircraft adhesive. The surface is first thoroughly cleaned using 200 proof ethanol and then the fiber is temporarily positioned using Kapton tape. Kapton tape is also used to lay down a masking line on either side of the fiber. A final cleaning of the surface is conducted with the ethanol immediately prior to application of the adhesive. The adhesive is applied in a layer approximately 0.5 to $1.0 \mathrm{~mm}$ thick, and care must be taken to roll the fiber back and forth to ensure the adhesive forms an effective bond between the fiber and the substrate.

\section{A. Beam Testing Procedure}

Validation of the shape estimation process using distributed strain measurements is conducted here on a cantilever beam. The material and geometric properties of the beam are as previously summarized in Table 1. For the static tests, the beam is instrumented with a single FOSS fiber on its lower surface oriented in the spanwise direction. The experimental setup and schematic for the static tests of the cantilever beam are shown in Fig. 11(a-b). Details of the fiber layout are summarized in Table 5; FBGs located in the section of the fiber which is looped back after reaching the tip are not used for analysis.

(a)
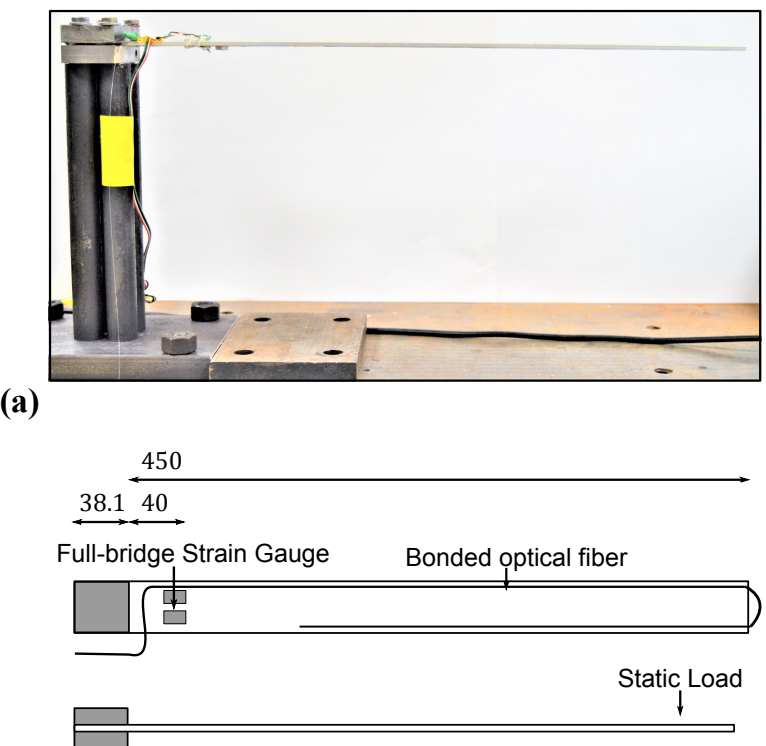

$\left.\right|_{\text {חリー }}$

(b)

Fig. 11 Experimental setups for the cantilever beam. (a) Static test setup of cantilever beam, (b) schematic of static test setup, (c) dynamic test setup of cantilever beam, and (d) schematic of dynamic test setup. Dimensions are in $\mathbf{m m}$. (c)
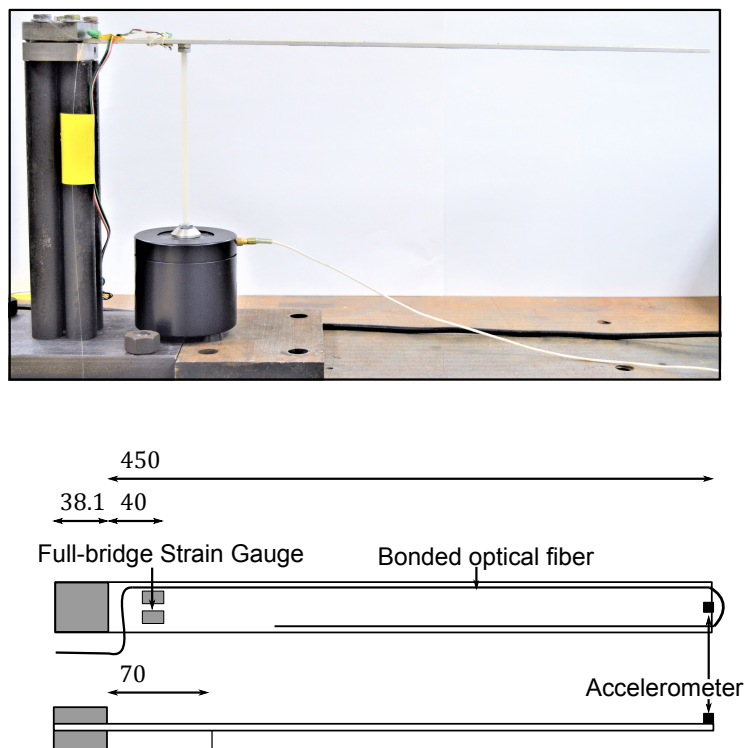

(d)

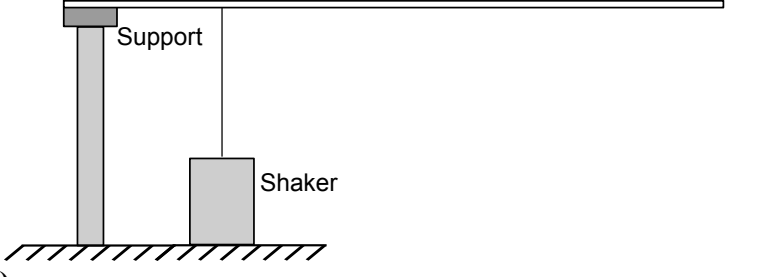

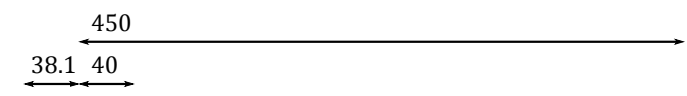


Table 5 Specifications of the fiber layout for the cantilever beam.

\begin{tabular}{ll}
\hline Parameter & Value $(\mathrm{mm})$ \\
\hline Spanwise locations of 22 FBGs & $x=20 i$ for $i=1,2, \ldots, 22$ \\
Chordwise distance from leading/trailing edge & 5 \\
\hline
\end{tabular}

A full-bridge strain gauge is also located on the beam at $40 \mathrm{~mm}$ from the root for calibration and comparison with the FOSS strain measurement located at the same position. During dynamic testing, the beam is excited by a shaker positioned on the beam centerline $70 \mathrm{~mm}$ from the beam root. The signal flow for these tests is given in Fig. 12.

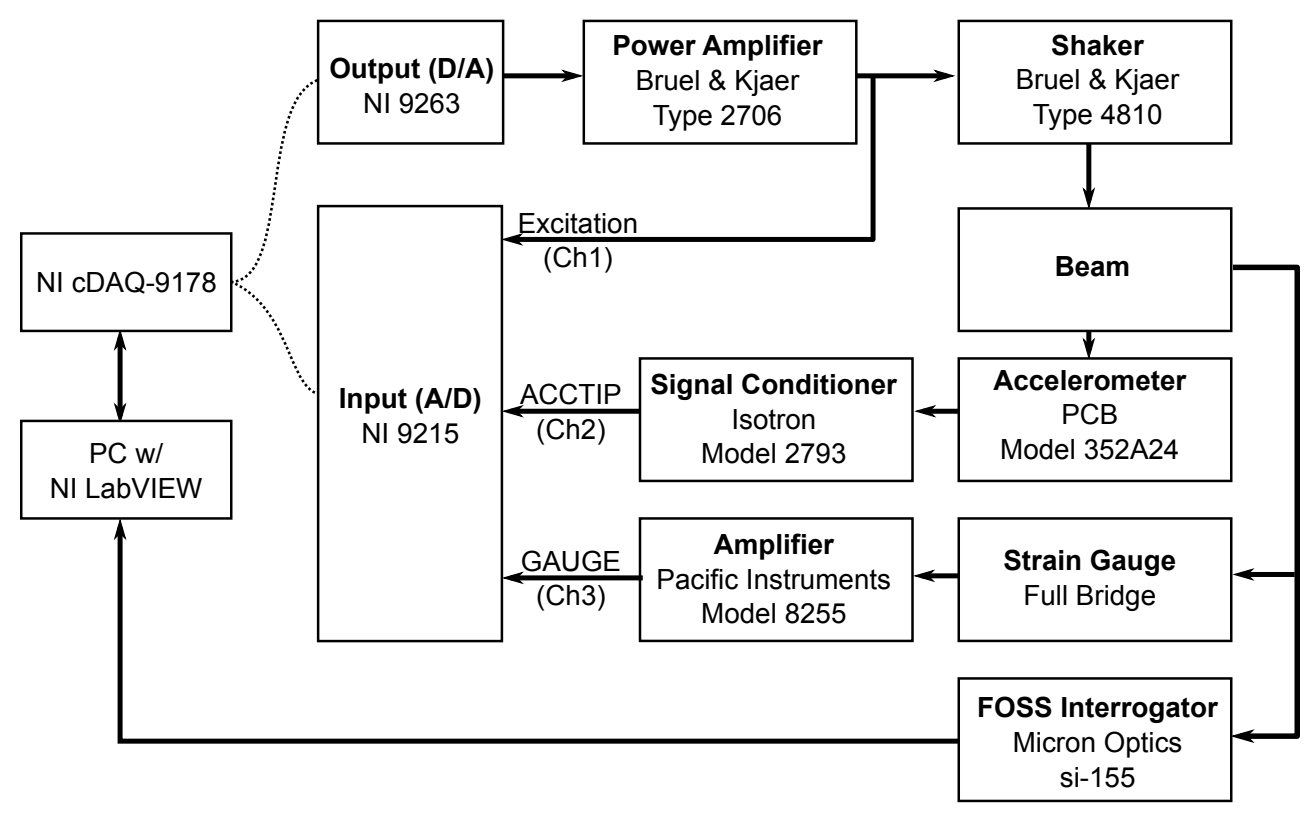

Fig. 12 Signal flow for experimental testing of the cantilever beam.

During static testing, loads are applied to the beam by a hanger and masses at $400 \mathrm{~mm}$ from the root. For the tip load results reported here, the $x$-coordinate is normalized by $400 \mathrm{~mm}$, i.e., $\hat{x}=1$ at $x=400$. This location is chosen in order to facilitate the application of static loads, which would be practically difficult to accomplish directly at the beam tip. Five different mass loads are applied: 100, 200, 300, 400, and $500 \mathrm{~g}$. Each increasing load is applied slowly and measurements taken after $60 \mathrm{~s}$ in order to allow any transient vibration to damp out. For each loading, FOSS and strain gauge measurements are taken for $10 \mathrm{~s}$ at a sampling rate of $1 \mathrm{kHz}$. The mean of these data for each sensor is then used as its static strain measurement in the analysis.

The nominal photoelastic coefficient for the FBGs, as reported by the fiber manufacturer FBGS, is $p_{e}=0.22 \varepsilon^{-1}$. To account for small differences in that sensitivity due to differential bonding location, adhesive, or manufacturing, the sensitivity of each FBG can be estimated by comparing its strain measurement with its corresponding analytical value for a given load case. This calibration process is completed for all five load cases and the sensitivity for each FBG estimated as the mean of these comparisons. Based on this method, the empirical photoelastic coefficients for the FBGs in this sequence of tests ranged from 0.19 to $0.22 \varepsilon^{-1}$, with a mean value of $0.22 \varepsilon^{-1}$.

For the dynamic tests, a sine sweep from 2 to $450 \mathrm{~Hz}$ with frequency steps of $0.1 \mathrm{~Hz}$ at a constant amplitude of excitation is conducted in order to determine the frequency response at each FBG. The experimental setup and schematic for the dynamic tests of the cantilever beam are shown in Fig. 11(c-d). At each frequency, 10 cycles of excitation are recorded at a sampling rate of $1 \mathrm{kHz}$. The standard deviation of this time data is then computed to yield an estimated amplitude of the strain response at each sensor for each frequency. The mean of the calibrated photoelastic coefficient, which is equal to the nominal value reported by the manufacturer $\left(p_{e}=0.22 \varepsilon^{-1}\right)$, is used for all FBGs in these tests. 


\section{B. Plate Testing Procedure}

Validation of the shape estimation process using distributed strain measurements is also conducted for a cantilever plate. The material and geometric properties of the rectangular plate are as previously summarized in Table 3. An image and schematic of the cantilever plate and fiber layout are shown in Fig. 13. The leading and trailing edge fibers are oriented spanwise to measure bending, and the midchord (twist rate) fiber is oriented in a 45-deg sawtooth pattern to measure shear strain. Details of the fiber layout are summarized in Table 6.

(a)
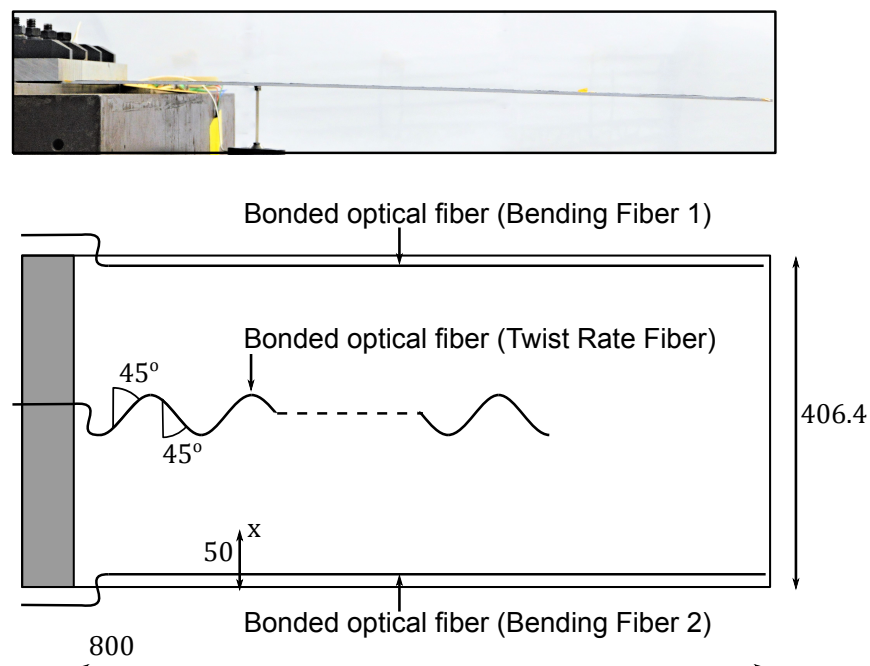

69200

Support

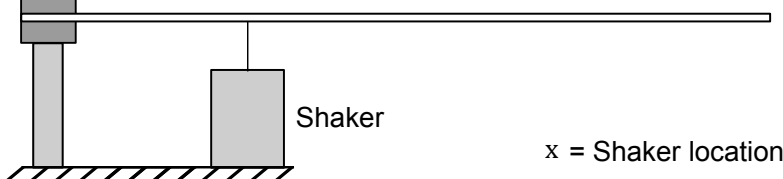

(b)

Fig. 13 Experimental setup for the cantilever plate. (a) Photograph and (b) schematic. Sawtooth pattern for the midchord twist rate fiber is not to scale. Dimensions are in $\mathbf{m m}$.

Table 6 Specifications of the fiber layout for the cantilever plate.

\begin{tabular}{lll}
\hline Fiber & Parameter & Value (mm) \\
\hline Bending Fibers 1 and 2 & Spanwise locations of 39 FBGs & $x=20 i$ for $i=1,2, \ldots, 39$ \\
& Chordwise distance from leading/trailing edge & 10 \\
Twist Rate Fiber & Spanwise locations of 39 FBGs & $x=15 i$ for $i=1,2, \ldots, 39$ \\
& Chordwise distance from leading/trailing edge & 203.2 \\
\hline
\end{tabular}

The plate is excited by a shaker positioned at $200 \mathrm{~mm}$ from the plate root and $50 \mathrm{~mm}$ from the trailing edge. The signal flow for these experiments is given in Fig. 14. 


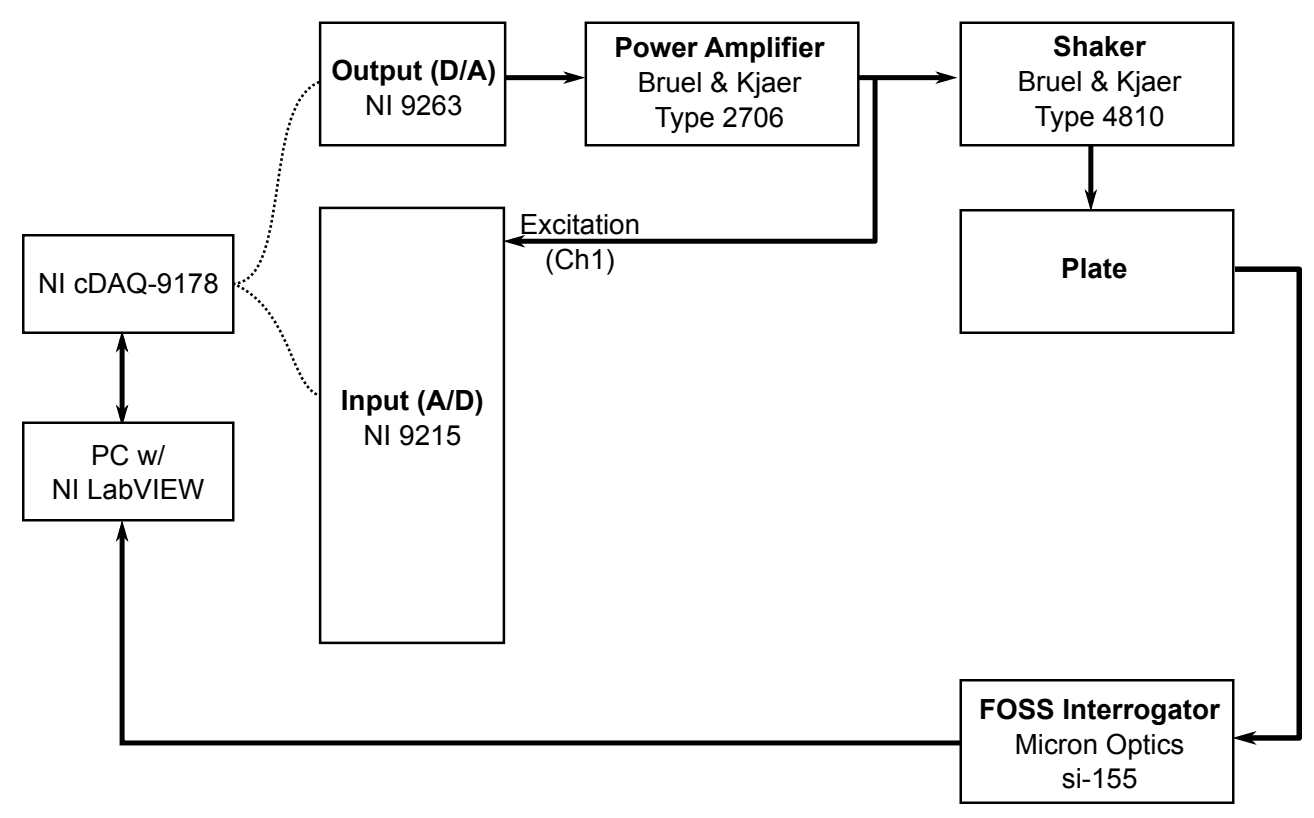

Fig. 14 Signal flow for experimental testing of the cantilever plate.

A sine sweep from 2 to $100 \mathrm{~Hz}$ with frequency steps of $0.1 \mathrm{~Hz}$ and at a constant amplitude of excitation is conducted in order to determine the strain frequency response along each fiber. At each frequency, 10 cycles of excitation are recorded at a sampling rate of $1 \mathrm{kHz}$. The standard deviation of this time data is then computed to yield an estimated amplitude of the strain response at each sensor for each frequency. The mean of the calibrated photoelastic coefficient, which is equal to the nominal value reported by the manufacturer $\left(p_{e}=0.22 \varepsilon^{-1}\right)$, is used for all FBGs in these tests.

\section{Beam Results}

In Fig. 15(a), the calculated curvature distribution based on the splined FOSS measurements is shown for each load case and compared with the calculated curvature based on the strain gauge as well as the analytical curvature based on the measured tip deflection. The analytical curvature profiles are determined by evaluating Eqn. 3 for the tip deflection $(\hat{x}=1)$, yielding the expression: $w(1)=Q l^{3} / 3 E I_{y}$. Substituting this relationship into Eqn. 4, the curvature distribution for a measured tip deflection, $w(1)$, is given by:

$$
\frac{d^{2} w(\hat{x})}{d \hat{x}^{2}}=3(1-\hat{x}) \cdot w(1)
$$

The splined FOSS data is integrated twice using the method described above and compared to the analytical deflection based on the measured tip deflection in Fig. 15(b). 

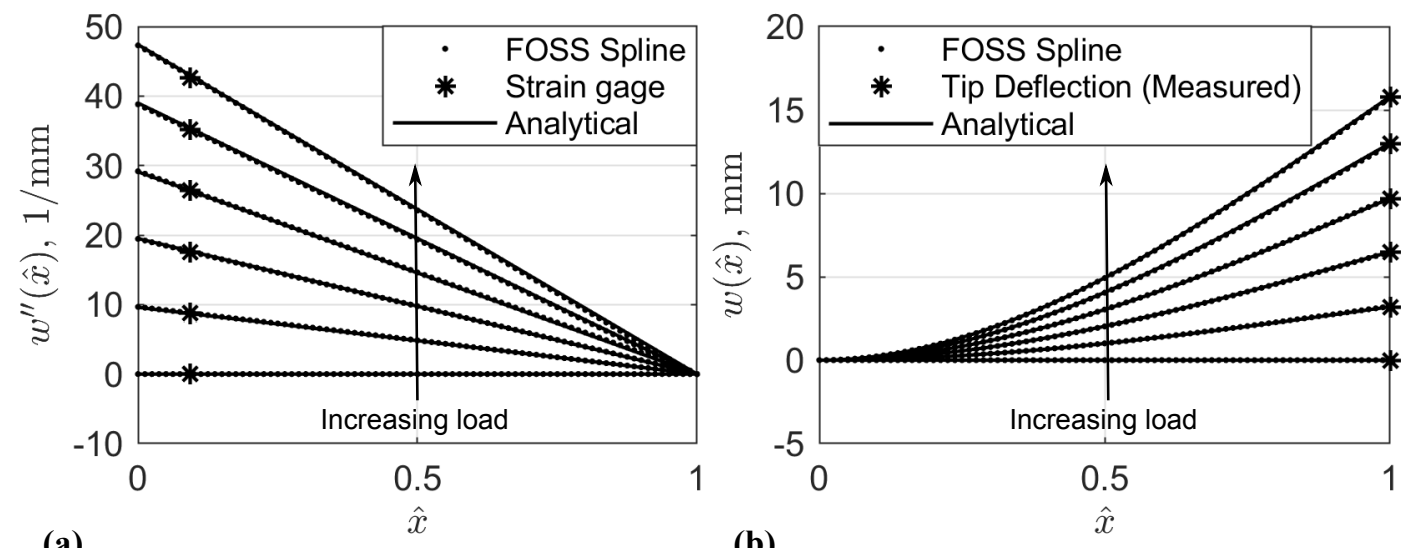

(a)

(b)

Fig. 15 (a) Curvature and (b) deflection of the cantilever beam based on integration of static experimental FOSS strain data for concentrated loads applied at $400 \mathrm{~mm}$.

The relative errors between the FOSS estimated and measured tip deflections are reported in Table 7.

Table 7 Error between FOSS estimated and measured static tip deflections.

\begin{tabular}{ccc}
\hline Load Case & Tip Mass $(g)$ & Error \\
\hline 1 & 100 & $-0.90 \%$ \\
2 & 200 & $0.18 \%$ \\
3 & 300 & $-0.30 \%$ \\
4 & 400 & $0.59 \%$ \\
5 & 500 & $0.43 \%$ \\
\hline
\end{tabular}

With errors less than $1 \%$ for all cases, the results based on the described integration method are relatively accurate even though strain measurements are only available at 20 locations during static testing. Using an OFDR system would likely provide even more accurate integrated results as less interpolation would be required to achieve high strain resolution throughout the beam.

In Fig. 16, the character of the strain distribution as a function of the frequency of excitation is illustrated, where the absolute value of the strain amplitude is plotted for each FBG. As the frequency increases from top to bottom, the absolute value of the strain at each sensor is plotted as a color map, with dark blue corresponding to low absolute strain and dark red to high absolute strain. The regions of high strain response occur near the resonant frequencies of the beam. Detail plots of the regions around the first four transverse bending resonant frequencies are shown in Fig. 17. 


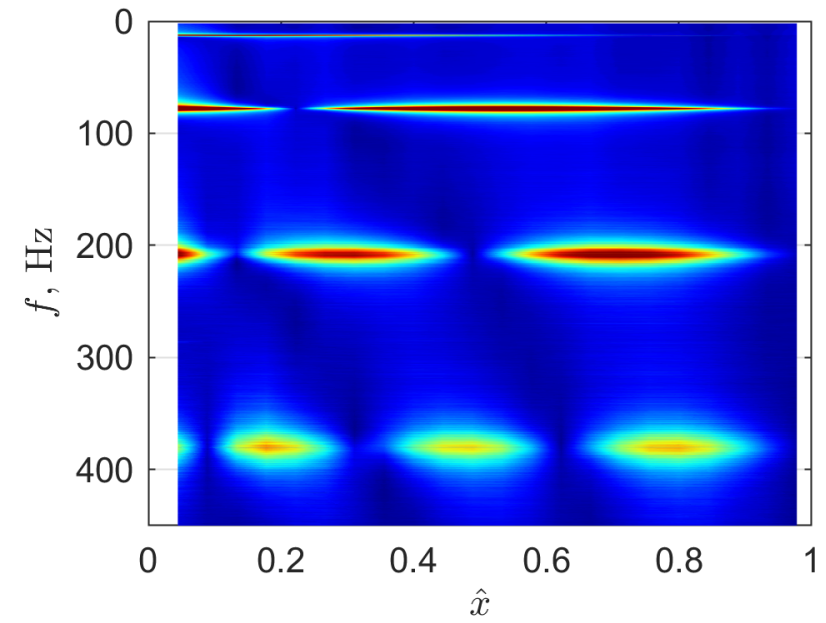

Fig. 16 Strain distribution in the cantilever beam subjected to harmonic excitation as a function of excitation frequency. Color map with dark blue/dark red corresponding to low/high absolute strain.

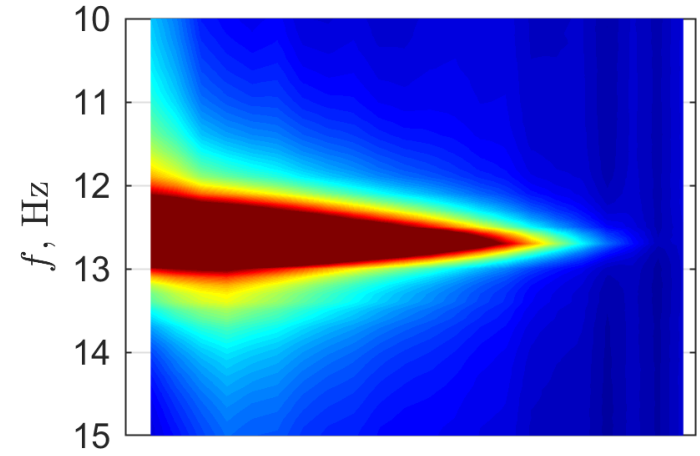

(a) Mode 1

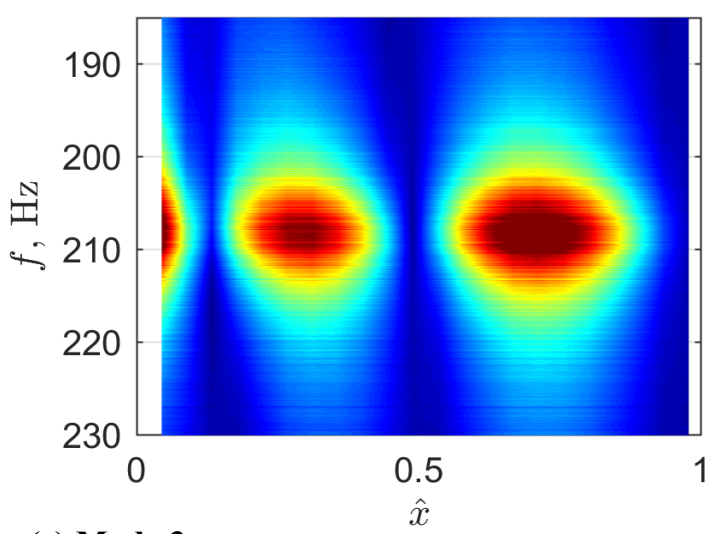

(c) Mode 3

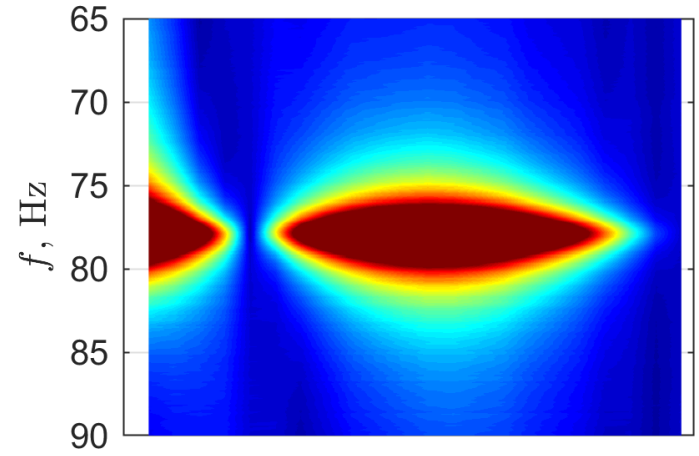

(b) Mode 2

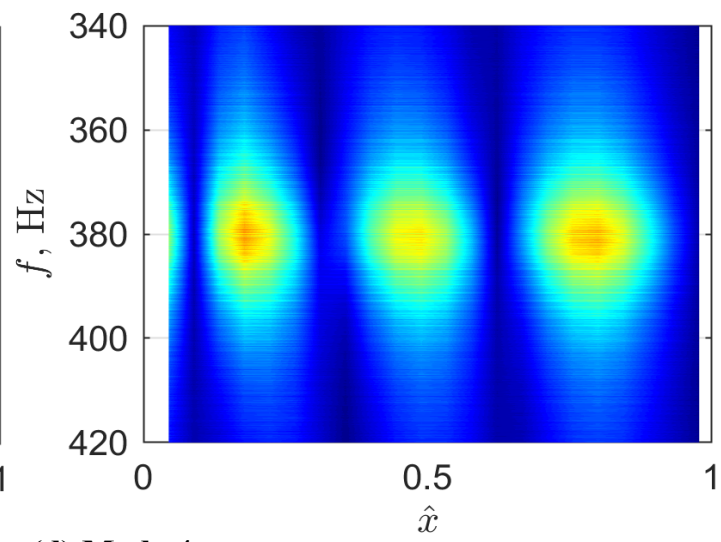

(d) Mode 4

Fig. 17 Detail of strain distribution in the cantilever beam subjected to harmonic excitation for the regions around the first four resonant frequencies. Color map with dark blue/dark red corresponding to low/high absolute strain.

In order to determine the experimental resonant frequencies of the beam, the summation of the absolute strain in all 
FBGs is calculated at each excitation frequency. This response metric, plotted in Fig. 18, is less noisy than the strain at any particular FBG and yields an improved estimate of the frequencies corresponding to maximum total strain response.

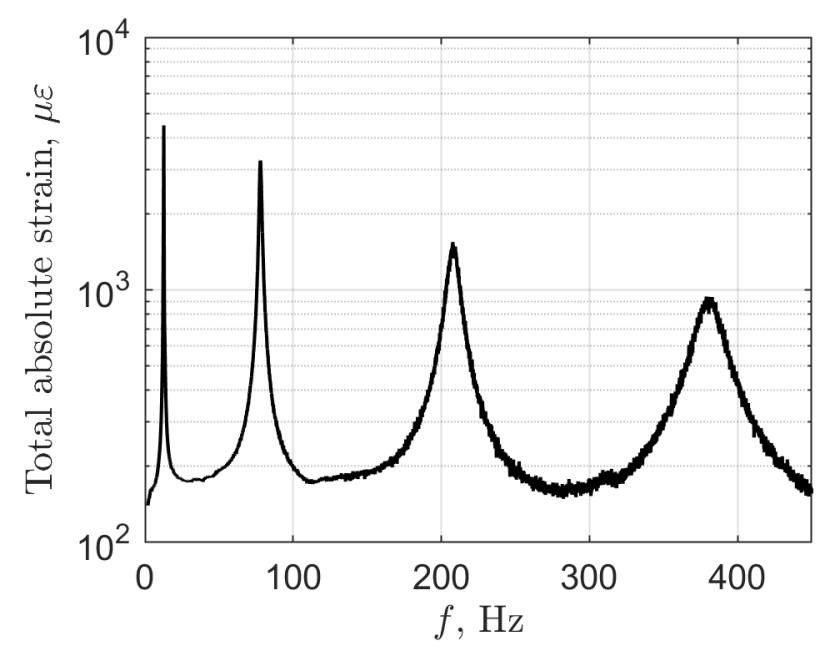

Fig. 18 Total measured strain in the cantilever beam as a function of excitation frequency.

The experimental resonant frequencies are then determined by choosing the four frequencies at which the total strain in the beam has a peak.

\section{Plate Results}

In Fig. 19, the character of the strain distribution as a function of the frequency of excitation is illustrated for the cantilever plate, where the absolute value of the strain amplitude is plotted for each FBG along each fiber. Similar to figures in the beam section, the absolute value of the strain at each sensor is plotted as a color map, with dark blue corresponding to low absolute strain and dark red to high absolute strain. The regions of high strain response occur near the natural frequencies of the plate. Detail plots of the regions around the first five resonant frequencies are shown in Fig. 20.

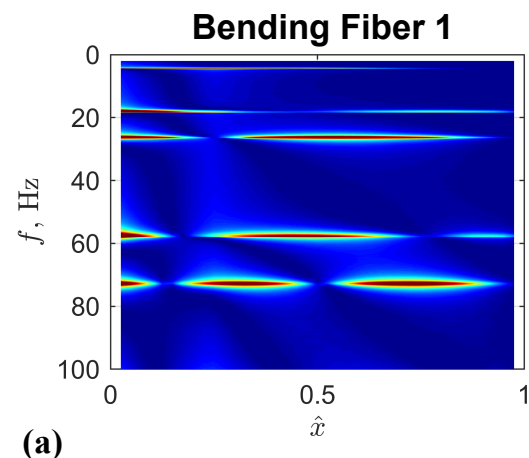

(a)

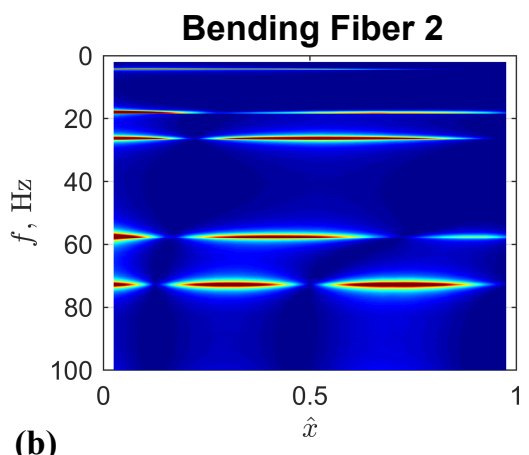

(b)

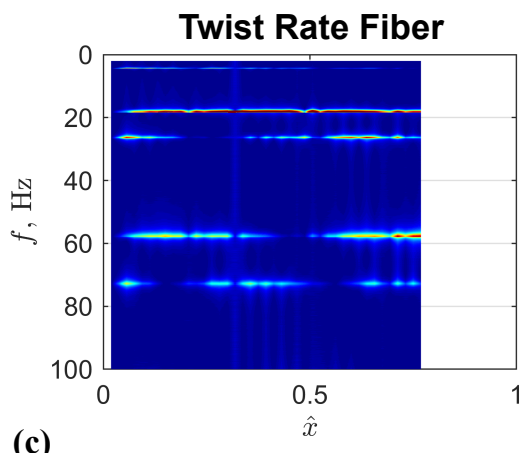

(c)

Fig. 19 Strain distribution in the cantilever plate subjected to harmonic excitation as a function of excitation frequency. (a) Leading edge (bending) fiber. (b) Trailing edge (bending) fiber. (c) Midchord (twist rate) fibers. Color map with dark blue/dark red corresponding to low/high strain absolute value. 


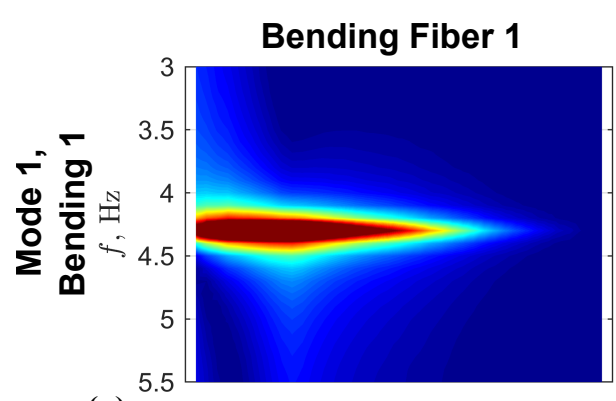

(a)

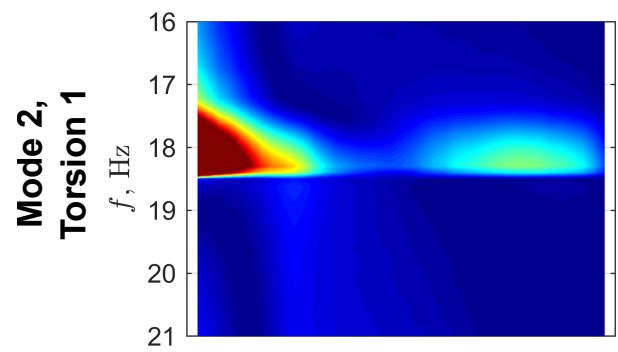

(d)

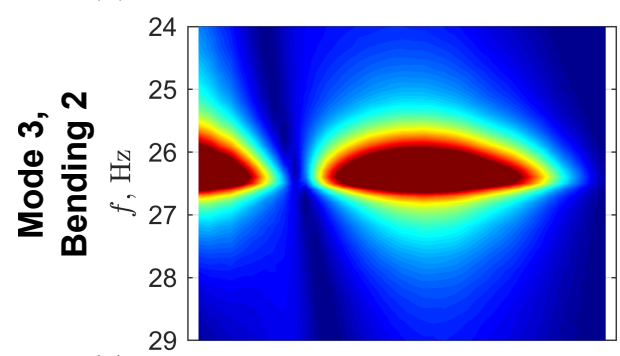

(g)

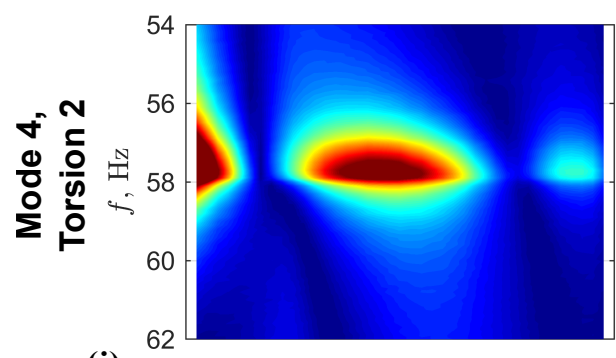

(j)

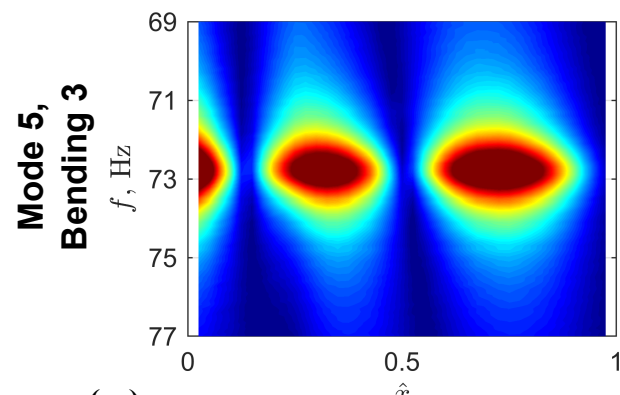

(m) (b)
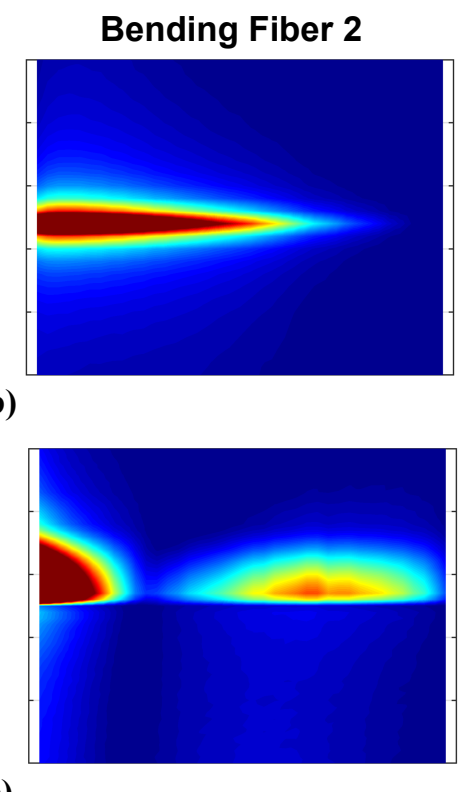

(e)

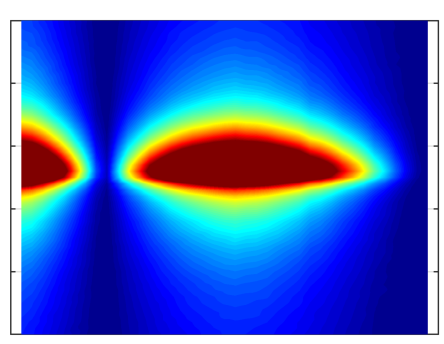

(h)

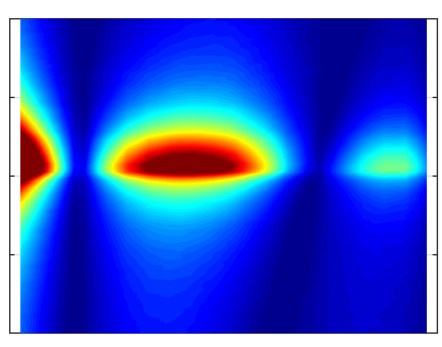

(k)

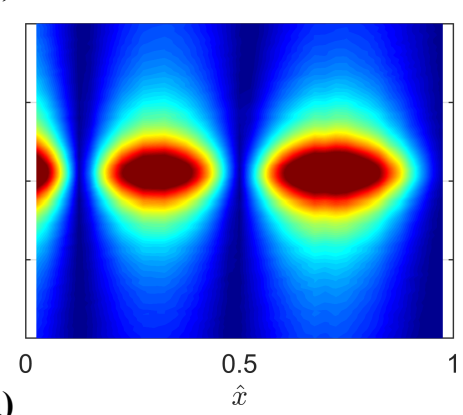

Twist Rate Fiber

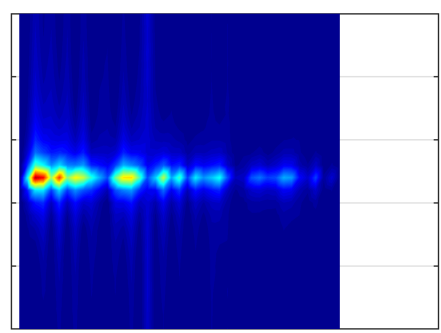

(c)

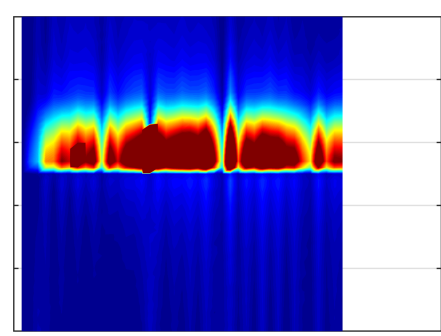

(f)

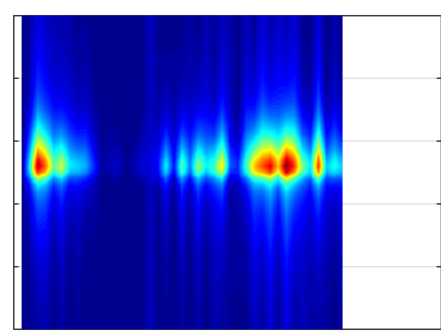

(i)

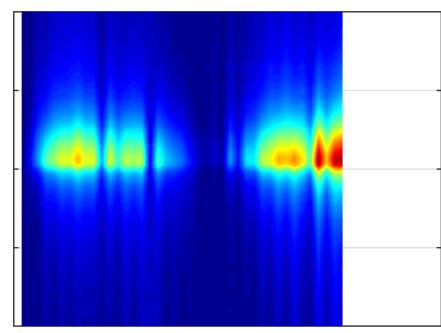

(I)

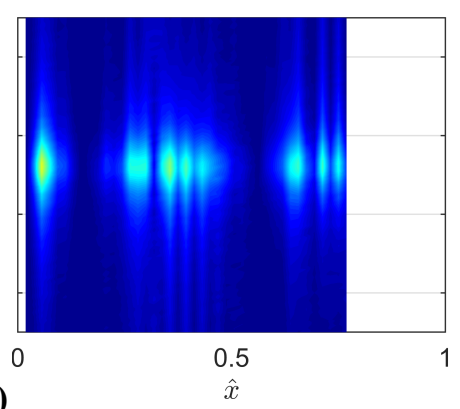

Fig. 20 Detail of strain distribution in the cantilever plate subjected to harmonic excitation for the regions around the first five resonant frequencies. Columns correspond to the leading edge, trailing edge, and midchord fibers, while rows correspond to the modes. Color map with dark blue/dark red corresponding to low/high strain absolute value. 
Although 39 FBGs are bonded in each of the fiber runs, the twist rate fiber sensors are positioned every $15 \mathrm{~mm}$, and so span from 15 to $585 \mathrm{~mm}$ along the plate's total length of $800 \mathrm{~mm}$. Upon visual inspection of the color map plots, it can be seen that the regions of high strain responses in each fiber correspond to the same frequency regions. In addition, although only a portion of the entire plate's length is covered with the twist rate fiber, this fiber has the largest response at the torsional modes.

In order to determine the experimental resonant frequencies of the plate, the total strain in each fiber is calculated at each excitation frequency. This response metric is plotted in Fig. 21 for each fiber.

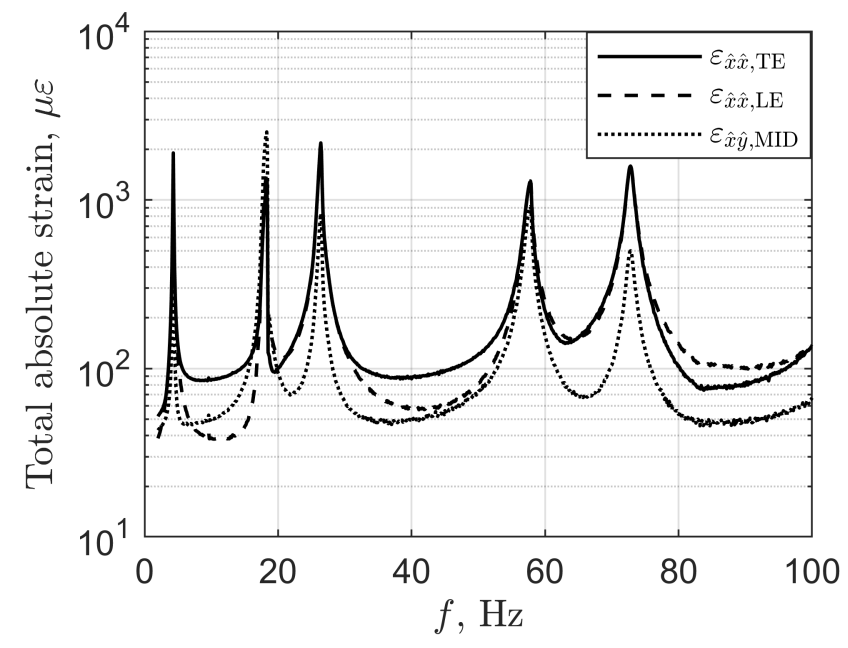

Fig. 21 Total measured strain in the cantilever plate for the three fibers as a function of excitation frequency.

The experimental resonant frequencies are then determined by choosing the five frequencies at which the total strain in one of the fibers has a peak. The peaks for all fibers are located at the same frequencies.

\section{Comparison of Theoretical and Experimental Results}

\section{A. Beam Comparison}

The normalized FOSS curvature splines at the identified natural frequencies are plotted in Fig. 22(a) along with the analytical curvature shapes. The normalization of the experimental data is conducted by adjusting a scaling factor to minimize the least squares residual between the analytical and scaled experimental curvature shapes. The reason for this scaling is to directly compare the shapes of the curvature distributions. The FOSS spline is then integrated twice to yield the normalized mode shapes, which are plotted with the analytical mode shapes in Fig. 22(b). 

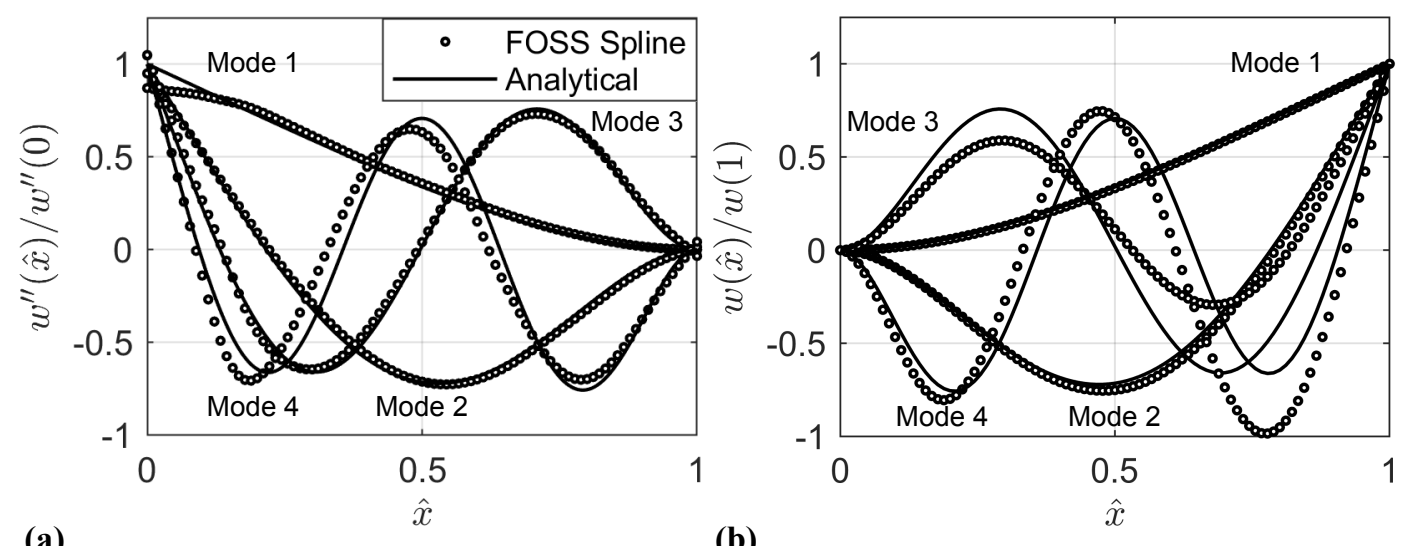

(b)

Fig. 22 (a) Curvature and (b) mode shapes of the cantilever beam based on integration of experimental FOSS strain data.

The curvature distributions based on FOSS agree relatively well with the analytical functions, although the accuracy does decrease as the mode number increases. The integrated mode shapes follow the same pattern, where the differences in shape increase for higher modes. These differences may be attributable to physical differences between the experiment and the analytical model or to experimental uncertainties, but the relative accuracy of these methods in comparison to the results of classical beam theory, particularly for the first two modes, is notable.

Although the torsion and in-plane bending modes are not estimated experimentally due to the transverse method of excitation, the different analytical predictions will be compared here for the beam properties given in Table 1 . The torsional constant for a rectangular section, based on the Fourier series solution to the exact torsion problem including warping, is found to be [36]:

$$
J=\frac{b h^{3}}{3}\left[1-\frac{192}{\pi^{5}} \frac{h}{b} \sum_{i=1,3,5, \ldots}^{\infty} \frac{1}{i^{5}} \tanh j \pi \frac{b}{2 h}\right] .
$$

Evaluating 10 terms in the series results in a torsional constant for the beam specimen of $J=394.562 \mathrm{~mm}^{4}$. It can then be shown that the $r$-th torsional natural frequency is [36]:

$$
\omega_{r}=\left(\sqrt{\frac{G J}{\rho I_{x} l^{2}}}\right)\left(\frac{(2 r-1) \pi}{2}\right)
$$

The analytical natural frequencies of the beam can be computed using the properties in Table 1 and the classical beam model previously described. The beam FE model was analyzed in MSC NASTRAN 2018.0 [33] using SOL 103 (normal modes analysis) and CQUAD4 elements. The mesh was refined until convergence in the reported natural frequencies to three decimal places. These values and those determined experimentally are summarized in Table 8 .

Table 8 Comparison of theoretical and experimental resonant frequencies for the cantilever beam (Hz). Theoretical models do not include damping.

\begin{tabular}{lcccccc}
\hline Method & $\begin{array}{c}\text { Mode 1 } \\
\text { (Bending 1) }\end{array}$ & $\begin{array}{c}\text { Mode 2 } \\
\text { (Bending 2) }\end{array}$ & $\begin{array}{c}\text { Mode 3 } \\
\text { (Bending 3) }\end{array}$ & $\begin{array}{c}\text { Mode 4 } \\
\text { (Bending 4) }\end{array}$ & $\begin{array}{c}\text { Mode 5 } \\
\text { (Torsion 1) }\end{array}$ & $\begin{array}{c}\text { Mode 6 } \\
\text { (Bending 5) }\end{array}$ \\
\hline Analytical & 13.0 & 81.7 & 155.4 & 228.8 & 281.1 & 448.4 \\
FE & 13.1 & 82.4 & 154.6 & 230.8 & 296.5 & 453.0 \\
Experimental & 12.7 & 77.8 & - & 207.8 & - & 380.5 \\
\hline
\end{tabular}

All bending modes reported in Table 8 are out-of-plane bending modes (i.e., bending about the $y$ axis shown in Fig. 1) except for the "Bending 3" mode, which is an in-plane bending mode (i.e., bending about the $z$ axis). The analytical 
estimate for this in-plane bending mode is determined by switching the role of the cross-sectional width and height in the computation of its second moment of area.

Although there is a general agreement between the theoretical and experimental values, the relative difference between them increases as the mode number increases. The influence of damping on the experimental values, which is not accounted for in the theoretical models (analytical and FE), is a contributing factor to these increasing differences. In addition to the increasing structural and air damping as the excitation frequency increases, the impact of experimental conditions (e.g., unmodeled mass/stiffness contributions from shaker/sensors/boundary conditions) increases for higher excitation frequencies.

\section{B. Plate Comparison}

The normalized strain shapes at the identified natural frequencies are plotted in Fig. 23 in comparison to the FE strain for the first four modes (originally shown in Fig. 10).

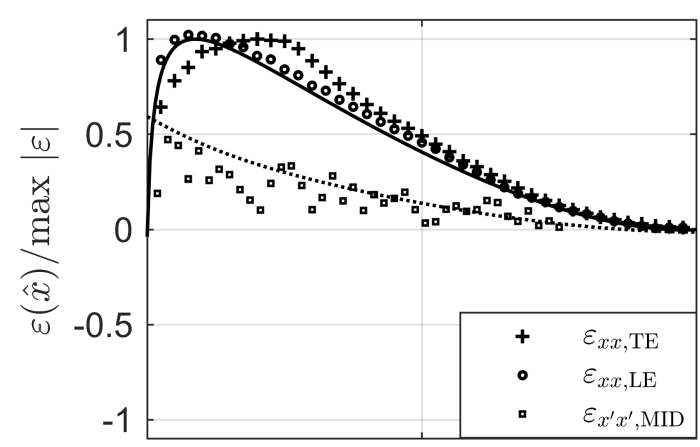

(a) Mode 1, Bending 1

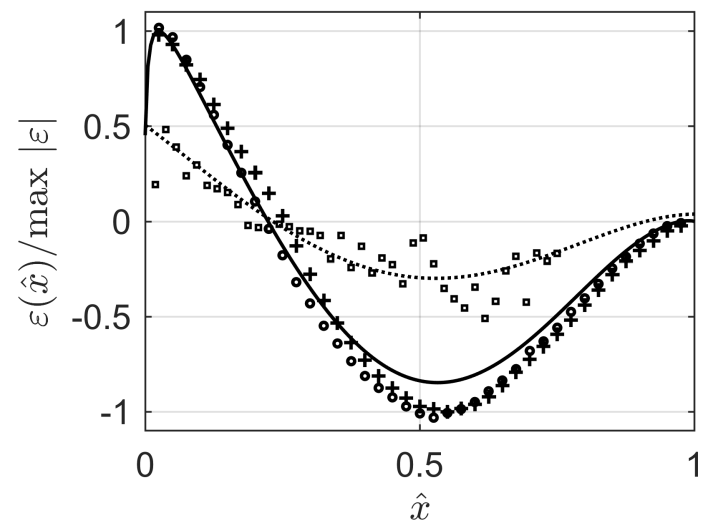

(c) Mode 3, Bending 2

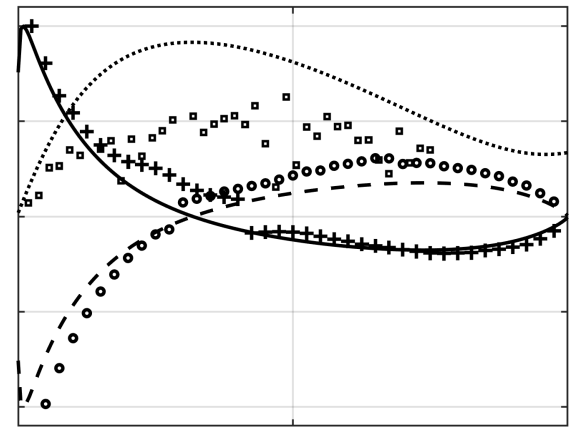

(b) Mode 2, Torsion 1

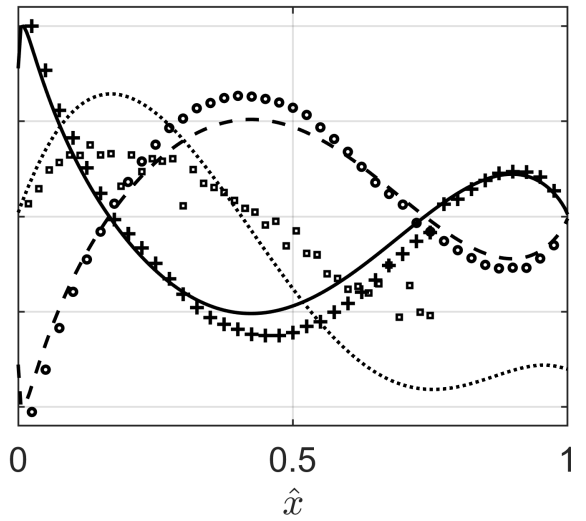

(d) Mode 4, Torsion 2

Fig. 23 Measured strain distribution in three fibers at the first four natural frequencies of a cantilever plate in comparison to $\mathrm{FE}$ results.

The experimental results for the bending fibers show relatively high correspondence with the strain distributions predicted through FE analysis for all four modes. The twist rate fiber results, on the other hand, generally follow the expected distribution based on FE analysis but are significantly more noisy.

Due to the noisy twist rate measurements, a method utilizing the leading and trailing edge bending fibers to estimate the mode shape is used here. Based on this method, the bending fibers will be taken to approximate the bending of thin strips at their respective edges, and then a surface between the two used to describe the deflection at interior points of the plate. Since only two bending fibers are available, a linear relationship is used to describe the connection between the bending lines:

$$
w(\hat{x}, \hat{y})=\hat{y} \cdot w_{\mathrm{LE}}(\hat{x})+(1-\hat{y}) \cdot w_{\mathrm{TE}}(\hat{x})
$$


where $w_{\mathrm{LE}}(\hat{x})$ and $w_{\mathrm{TE}}(\hat{x})$ are the bending deflections at the leading and trailing edges calculated using the integration matrix method described above for beams. Comparisons between these calculated mode shapes and those based on FE analysis/products of beam functions are shown in Fig. 24.

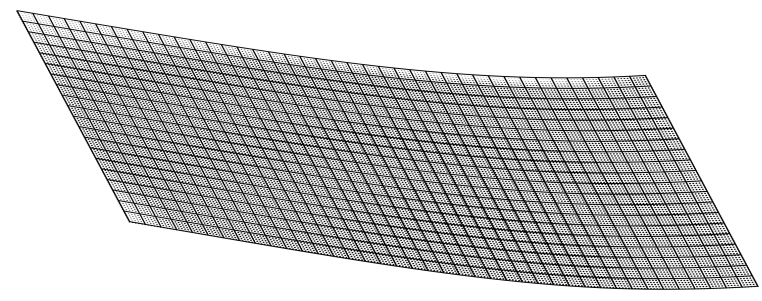

(a) Mode 1, Bending 1

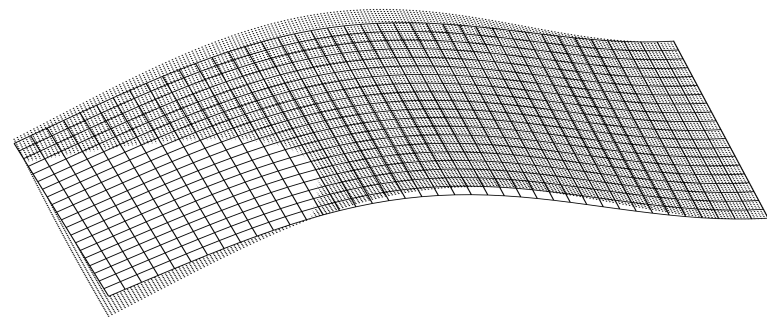

(c) Mode 3, Bending 2

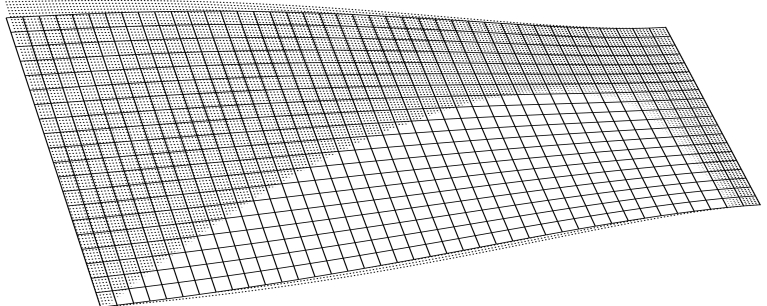

(b) Mode 2, Torsion 1

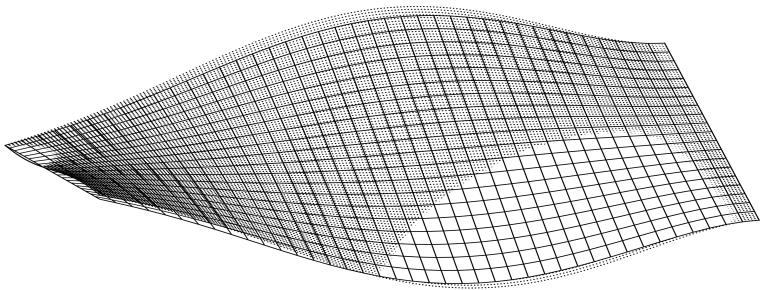

(d) Mode 4, Torsion 2

Fig. 24 Comparison of plate mode shapes determined experimentally (grids) and by FE analysis (dots) for the first four modes.

Although there are noticeable differences between the FOSS estimated shapes and the numerical models, the FOSS integration method based on measured strain is relatively accurate in comparison. In addition to experimental uncertainties, a portion of these differences may be attributable to physical differences between the experiment and the analytical models (e.g., the effect of the shaker).

Using the natural frequency parameters reported in Table 4, which are based on the Rayleigh-Ritz method and modes shapes approximated with products of beam functions, the natural frequency predictions of the plate can be determined by Eqn. (18). The natural frequencies based on these frequency parameters, FE analysis, and those determined experimentally are summarized in Table 9.

Table 9 Comparison of theoretical and experimental resonant frequencies for the cantilever plate (Hz). Theoretical models do not include damping.

\begin{tabular}{lccccc}
\hline Method & $\begin{array}{c}\text { Mode 1 } \\
\text { (Bending 1) }\end{array}$ & $\begin{array}{c}\text { Mode 2 } \\
\text { (Torsion 1) }\end{array}$ & $\begin{array}{c}\text { Mode 3 } \\
\text { (Bending 2) }\end{array}$ & $\begin{array}{c}\text { Mode 4 } \\
\text { (Torsion 2) }\end{array}$ & $\begin{array}{c}\text { Mode 5 } \\
\text { (Bending 3) }\end{array}$ \\
\hline Rayleigh-Ritz & 4.2 & 17.5 & 26.3 & 57.2 & 73.7 \\
FE & 4.2 & 17.4 & 26.1 & 57.1 & 73.3 \\
Experimental & 4.3 & 18.3 & 26.4 & 57.8 & 72.8 \\
\hline
\end{tabular}

The experimental resonant frequencies agree well with the numerical predictions (Rayleigh-Ritz and FE). Damping, imperfect boundary conditions, and unmodeled contributions from the shaker/fiber bonds are all potential sources of experimental differences.

\section{Conclusion}

In this paper, the feasibility of using FOSS technology for real-time static and dynamic shape sensing of flexible bodies is investigated. Strain-based methods for shape estimation are described and then applied to experimental strain 
data for a cantilever beam and plate. In comparison to theoretical models of these systems, it is shown that FOSS measurements can be used to accurately estimate the static and dynamic shapes of the benchtop structures and estimate their resonant frequencies.

Based on these experiments, the next stage of this research will be the validation of FOSS feedback control in benchtop testing. One of the key capabilities of FOSS is low-mass/low-volume distributed sensing, which may enable improved controller performance for a variety of control objectives (e.g., quasistatic shape control or modal suppression). Follow-on work will then focus on the application of this sensing technology to the IAWTM aeroelastic model to be tested in the TDT.

\section{Acknowledgments}

This work is funded by the NASA Advanced Air Transport Technology (AATT) Project.

\section{References}

[1] Ajaj, R., Friswell, M., and Saavedra Flores, E., "On the effectiveness of active aeroelastic structures for morphing aircraft," Aeronautical Journal, Vol. 117, No. 1197, 2013, pp. 1165-1174.

[2] Florance, J. P., Burner, A. W., Fleming, G. A., Hunter, C. A., Graves, S. S., and Martin, C. A., "Contributions of the NASA Langley Research Center to the DARPA/AFRL/NASA/Northrop Grumman Smart Wing Program," Proceedings of the AIAA Dynamics Specialists Conference, Norfolk, VA, 2003.

[3] Heeg, J., Spain, C. V., Florance, J. R., Wieseman, C. D., Ivanco, T. G., DeMoss, J. A., Silva, W. A., Panetta, A., Lively, P., and Tumwa, V., "Experimental Results from the Active Aeroelastic Wing Wind Tunnel Test Program," 46th AIAA/ASME/ASCE/AHS/ASC Structures, Structural Dynamics and Materials Conference, 2005.

[4] Ivanco, T., Scott, R., Love, M., Zink, S., and Weisshaar, T., "Validation of the Lockheed Martin morphing concept with wind tunnel testing," 48th AIAA/ASME/ASCE/AHS/ASC Structures, Structural Dynamics, and Materials Conferences, 2007.

[5] Pendleton, E. W., Bessette, D., Field, P. B., Miller, G. D., and Griffin, K. E., "Active aeroelastic wing flight research program: technical program and model analytical development," Journal of Aircraft, Vol. 37, No. 4, 2000, pp. 554-561.

[6] Woods-Vedeler, J. A., Pototzky, A. S., and Hoadley, S. T., "Active Load Control During Rolling Maneuvers," Tech. rep., NASA/TP-3455, 1994.

[7] Florance, J., Heeg, J., Spain, C., Ivanco, T., Wieseman, C., and Lively, P., "Variable stiffness spar wind-tunnel model development and testing," 45th AIAA/ASME/ASCE/AHS/ASC Structures, Structural Dynamics \& Materials Conference, 2004.

[8] Pendleton, E., Flick, P., Paul, D., Voracek, D., Reichenbach, E., and Griffin, K., "The X-53 A summary of the active aeroelastic wing flight research program," 48th AIAA/ASME/ASCE/AHS/ASC Structures, Structural Dynamics, and Materials Conference, 2007, p. 1855.

[9] Perry III, B., Cole, S. R., and Miller, G. D., "Summary of an active flexible wing program,” Journal of Aircraft, Vol. 32, No. 1, 1995, pp. 10-15.

[10] Ko, W. L., Richards, W. L., and Tran, V. T., "Displacement Theories for In-Flight Deformed Shape Predictions of Aerospace Structures," NASA/TP-2007-214612, 2007.

[11] Vazquez, S. L., Tessler, A., Quach, C. C., Cooper, E. G., Parks, J., and Spangler, J. L., "Structural Health Monitoring Using High-Density Fiber Optic Strain Sensor and Inverse Finite Element Methods,” Tech. rep., NASA/TM-2005-213761, 2005.

[12] Gherlone, M., Cerracchio, P., Mattone, M., Sciuva, M. D., and Tessler, A., "Shape sensing of 3D frame structures using and inverse Finite Element Method,” International Journal of Solids and Structures, Vol. 49, 2012, pp. 3100-3112.

[13] Pak, C., “Wing Shape Sensing from Measured Strain,” AIAA Journal, Vol. 54, No. 3, 2016, pp. 1064-1073.

[14] Jones, R. T., Bellemore, D. G., Berkoff, T. A., Sirkis, J. S., Davis, M. A., Putnam, M. A., Friebele, E. J., and Kersey, A. D., "Determination of cantilever plate shapes using wavelength division multiplexed fiber Bragg grating sensors and a least-squares strain-fitting algorithm,” Smart Materials and Structures, Vol. 7, 1998, pp. 178-188.

[15] Jutte, C. V., Ko, W. L., Stephens, C. A., Bakalyar, J. A., Richards, W. L., and Parker, A. R., "Deformed Shape Calculation of a Full-Scale Wing Using Fiber Optic Strain Data from a Ground Loads Test,” Tech. rep., NASA/TP-2011-215975, 2011. 
[16] Glaser, R., Caccese, V., and Shahinpoor, M., "Shape Monitoring of a Beam Structure from Measured Strain or Curvature," Experimental Mechanics, Vol. 52, No. 6, 2012, pp. 591-606.

[17] Bakalyar, J. A., and Jutte, C., "Validation Tests of Fiber Optic Strain-Based Operational Shape and Load Measurements," AIAA Structures, Structural Dynamics and Materials Conference, 23-26 April 2012, Honolulu, Hawaii, 2012.

[18] Derkevorkian, A., Masri, S. F., Alvarenga, J., Boussalis, H., Bakalyar, J., and Richards, W. L., "Strain-Based Deformation Shape-Estimation Algorithm for Control and Monitoring Applications,” AIAA Journal, Vol. 51, No. 9, 2013, pp. 2231-2240.

[19] Nicolas, M. J., Sullivan, R. W., and Richards, W. L., "Large Scale Applications Using FBG Sensors: Determination of In-Flight Loads and Shape of a Composite Aircraft Wing," Aerospace, Vol. 3, No. 18, 2016.

[20] Cusano, A., Capoluongo, P., Campopiano, S., Cutolo, A., Giordano, M., Felli, F., Paolozzi, A., and Caponero, M., "Experimental Modal Analysis of an Aircraft Model Wing by Embedded Fiber Bragg Grating Sensors," IEEE Sensors Journal, Vol. 6, No. 1, 2006, pp. 67-77.

[21] Jiang, H., van der Veek, B., Kirk, D., and Gutierrez, H., "Real-Time Estimation of Time-Varying Bending Modes Using Fiber Bragg Grating Sensor Arrays,” AIAA Journal, Vol. 51, No. 1, 2013, pp. 178-185.

[22] Braghin, F., Cazzulani, G., Cinquemani, S., and Resta, F., "Potential of FBG Sensors for Vibration Control in Smart Structures," Proceedings of the 2013 IEEE International Conference on Mechatronics, Vicenza, Italy, 2013.

[23] Cazzulani, G., Cinquemani, S., Comolli, L., and Resta, F., "A quasi-modal approach to overcome FBG limitations in vibration control of smart structures," Smart Materials and Structures, Vol. 22, 2013.

[24] Cazzulani, G., Cinquemani, S., Comolli, L., Gardella, A., and Resta, F., "Vibration control of smart structures using an array of Fiber Bragg Grating sensors," Mechatronics, Vol. 24, 2014, pp. 345-353.

[25] Vassberg, J. C., DeHaan, M. A., Rivers, S. M., and Wahls, R. A., "Development of a Common Research Model for Applied CFD Validation Studies," 26th AIAA Applied Aerodynamics Conference, 2008.

[26] Craig, R. R., and Kurdila, A. J., Fundamentals of structural dynamics, $2^{\text {nd }}$ ed., John Wiley \& Sons, Inc., 2006.

[27] Hibbeler, R. C., Engineering Mechanics. Statics., 13 ${ }^{\text {th }}$ ed., Pearson, Upper Saddle River, N.J., 2013.

[28] Shames, I. H., and Dym, C. L., Energy and Finite Element Methods in Structural Mechanics, Taylor \& Francis, 2003.

[29] Warburton, G., “The Vibration of Rectangular Plates," Proceedings of the Institution of Mechanical Engineers, Vol. 168, No. 1, 1954, pp. 371-384.

[30] Leissa, A. W., "Vibration of Plates," NASA SP-160, National Aeronautics and Space Administration, 1969.

[31] Leissa, A., “The Free Vibration of Rectangular Plates," Journal of Sound and Vibration, Vol. 31, No. 3, 1973, pp. $257-293$.

[32] Bhat, R. B., "Natural Frequencies of Rectangular Plates using Characteristic Orthogonal Polynomials in Rayleigh-Ritz Method," Journal of Vibration and Sound, Vol. 102, No. 4, 1985, pp. 493-499.

[33] MSC, “MSC Nastran 2018.0,” software, 2018.

[34] Tessler, A., and Spangler, J. L., "A least-squares variational method for full-field reconstruction of elastic deformations in shear-deformable plates and shells," Computer methods in applied mechanics and engineering, Vol. 194, No. 2-5, 2005, pp. $327-339$

[35] Sadd, M. H., Elasticity: Theory, Applications, and Numerics, Elsevier, 2005.

[36] Rao, S. S., Vibration of Continuous Systems, John Wiley \& Sons, Inc., 2007. 